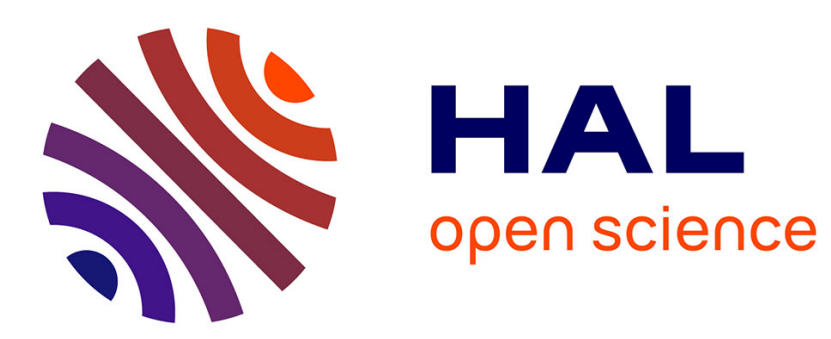

\title{
Intertwining operators associated to a family of differential-reflection operators
}

Salem Ben Said, Asma Boussen, Mohamed Sifi

\section{To cite this version:}

Salem Ben Said, Asma Boussen, Mohamed Sifi. Intertwining operators associated to a family of differential-reflection operators. 2016. hal-01282498

\section{HAL Id: hal-01282498 \\ https://hal.science/hal-01282498}

Preprint submitted on 3 Mar 2016

HAL is a multi-disciplinary open access archive for the deposit and dissemination of scientific research documents, whether they are published or not. The documents may come from teaching and research institutions in France or abroad, or from public or private research centers.
L'archive ouverte pluridisciplinaire HAL, est destinée au dépôt et à la diffusion de documents scientifiques de niveau recherche, publiés ou non, émanant des établissements d'enseignement et de recherche français ou étrangers, des laboratoires publics ou privés. 


\title{
INTERTWINING OPERATORS ASSOCIATED TO A FAMILY OF DIFFERENTIAL-REFLECTION OPERATORS
}

\author{
SALEM BEN SAID, ASMA BOUSSEN \& MOHAMED SIFI
}

\begin{abstract}
We introduce a family of differential-reflection operators $\Lambda_{A, \varepsilon}$ acting on smooth functions defined on $\mathbb{R}$. Here $A$ is a Sturm-Liouville function with additional hypotheses and $\varepsilon \in \mathbb{R}$. For special pairs $(A, \varepsilon)$, we recover Dunkl's, Heckman's and Cherednik's operators (in one dimension). The spectral problem for the operators $\Lambda_{A, \varepsilon}$ is studied. In particular, we obtain suitable growth estimates for the eigenfunctions of $\Lambda_{A, \varepsilon}$

As the operators $\Lambda_{A, \varepsilon}$ are mixture of $d / d x$ and reflection operators, we prove the existence of an intertwining operator $V_{A, \varepsilon}$ between $\Lambda_{A, \varepsilon}$ and the usual derivative. The positivity of $V_{A, \varepsilon}$ is also established.
\end{abstract}

2010 Mathematics Subject Classification. 34K99, 34B25, 33E30.

Keywords. Differential-reflection operators, spectral problem, intertwining operators.

\section{INTRODUCTION}

Dunkl's ascertainment in the late eighties of the operators that now bear his name is one of the most significant developments in the theory of special functions associated to root systems [9]. Some early work in this area was done by Koornwinder [20], Heckman and Opdam [18], and Opdam [23]. A lot of the motivation for the subject comes from analysis on Riemannian symmetric spaces.

In [9] Dunkl generalized the operator $\partial / \partial x_{i}$ to a mixture of a differential and a reflection operator. In one dimension it is denoted by $D_{\alpha}, \alpha \geq-1 / 2$. By the specialization $\alpha=\frac{1}{2} d-1$ with $d \in \mathbb{N}_{\geq 2}$, the operator $D_{\alpha}^{2}$ coincides on even functions with the radial part of the Laplace operator on the flat symmetric space $M(d) / S O(d)$, where $M(d)$ is the motion group of $\mathbb{R}^{d}$. Important work in the analysis of Dunkl operators has been done by several authors (see for instance [9, 11, 12, 16, 24, 29, 34]; this list is far from being complete).

Some years after, Heckman [17] wrote down a trigonometric variant of the Dunkl operators. In one dimension it is denoted by $H_{\alpha, \beta}, \alpha \geq \beta \geq-1 / 2, \alpha \neq-1 / 2$. For $\alpha=\frac{1}{2}(p-1)$ and $\beta=\frac{1}{2}(q-1)$ with $p \geq q>0$, the restriction of $H_{\alpha, \beta}^{2}$ to even functions coincides with the radial part of the Laplace-Beltrami operator on Riemannian symmetric spaces of the non-compact type and of real rank one. Significant results in the analysis of Heckman operators have been obtained by several authors (see for instance [2, 8, 19]).

Next, in [7] Cherednik made a slight but significant variation of Heckman's operator. See [16, 25, 35] for a comparison between Heckman and Cherednik operators.

In the present paper we consider some aspects of harmonic analysis associated to the following family of $(A, \varepsilon)$-operators

$$
\Lambda_{A, \varepsilon} f(x)=f^{\prime}(x)+\frac{A^{\prime}(x)}{A(x)}\left(\frac{f(x)-f(-x)}{2}\right)-\varepsilon \varrho f(-x),
$$

where $A$ is so-called a Chébli function on $\mathbb{R}$ (i.e. $A$ is a continuous $\mathbb{R}^{+}$-valued function on $\mathbb{R}$ satisfying certain regularity and convexity hypotheses), $\varrho$ is the index of $A$, and $\varepsilon \in \mathbb{R}$. We note 
that $\varrho \geq 0$. The function $A$ and the real number $\varepsilon$ are the deformation parameters giving back the above three cases (as special examples): Dunkl's operators when $A(x)=A_{\alpha}(x)=|x|^{2 \alpha+1}$ and $\varepsilon$ arbitrary; Heckman's operators when $A(x)=A_{\alpha, \beta}(x)=|\sinh x|^{2 \alpha+1}(\cosh x)^{2 \beta+1}$ and $\varepsilon=0$; and Cherednik's operators when $A(x)=A_{\alpha, \beta}(x)=|\sinh x|^{2 \alpha+1}(\cosh x)^{2 \beta+1}$ and $\varepsilon=1$.

We would like to mention that a differential-reflection operator built in terms of a Chébli function has been the first time done in [22]. This is one of the major themes of research at the "school of harmonic analysis" in Tunisia.

This paper consists of tow parts. In the first part we consider the spectral problem for this family of $(A, \varepsilon)$-operators (1.1). More precisely, let $\lambda \in \mathbb{C}$ and consider the equation

$$
\Lambda_{A, \varepsilon} f(x)=i \lambda f(x),
$$

where $f: \mathbb{R} \rightarrow \mathbb{C}$. We prove that there exists a unique solution $\Psi_{A, \varepsilon}(\lambda, \cdot)$ of (1.2) satisfying $\Psi_{A, \varepsilon}(\lambda, 0)=1$. Moreover, under the assumption $-1 \leq \varepsilon \leq 1$, we establish in Theorems 3.4 and 3.5 suitable estimates for the growth of the eigenfunction $\Psi_{A, \varepsilon}(\lambda, x)$ and of its partial derivatives. Our first step is Theorem 3.3 , where we prove that $\Psi_{A, \varepsilon}(\lambda, \cdot)>0$ whenever $\lambda \in i \mathbb{R}$. In a forthcoming paper [4], these estimates will play crucial tools for developing $L^{p}$-Fourier analysis associated to the $(A, \varepsilon)$-operators (1.1).

We note that $\Psi_{A, \varepsilon}$ reduces to the Dunkl kernel in the $\left(A_{\alpha}, \varepsilon\right)$-case [10, 26]; to the Heckman kernel in the $\left(A_{\alpha, \beta}, 0\right)$-case [2, 8, 19]; and to the Cherednik kernel (or Opdam's kernel) in the $\left(A_{\alpha, \beta}, 1\right)$-case [1, 14, 25].

In the second part of this paper we start by studying the existence of an intertwining operator between $\Lambda_{A, \varepsilon}$ and the ordinary derivative. We prove that there exists a unique isomorphism $V_{A, \varepsilon}: C^{\infty}(\mathbb{R}) \rightarrow C^{\infty}(\mathbb{R})$ satisfying $\Lambda_{A, \varepsilon} \circ V_{A, \varepsilon}=V_{A, \varepsilon} \circ \frac{d}{d x}$, with $V_{A, \varepsilon} f(0)=f(0)$ (see Theorem 4.7). The construction of $V_{A, \varepsilon}$ involves Delsarte type operators [21,31]. Our construction generalizes the one given in [13] for $A(x)=A_{\alpha, \beta}(x)=|\sinh x|^{2 \alpha+1}(\cosh x)^{2 \beta+1}$ and $\varepsilon=1$. For $A(x)=A_{\alpha}(x)=$ $|x|^{2 \alpha+1}$ and $\varepsilon$ arbitrary, the intertwining operator $V_{A, \varepsilon}$ reduces to the Dunkl intertwining operator in one dimension [28].

Another important result concerning the intertwining operator $V_{A, \varepsilon}$ is that the latter is of positive type in the sense that, if $f \geq 0$ then $V_{A, \varepsilon} f \geq 0$ (see Theorem 4.10). The major technical step in the proof of Theorem 4.10 is the positivity of $V_{A, \varepsilon}\left(h_{t}(u, \cdot)\right)(x)$, where $h_{t}(u, v)$ denotes the Euclidean heat kernel at time $t>0$. For $\varepsilon=0$ and 1, this result can be found in [36] and [37], while for $A(x)=A_{\alpha}(x)=|x|^{2 \alpha+1}$ and $\varepsilon$ arbitrary, the positivity of $V_{A, \varepsilon}$ goes back to [27]. We pin down that the positivity of $V_{A, \varepsilon}$ played a fundamental role in [3] in establishing an analogue of Beurling's theorem, and its relatives such as theorems of type Gelfand-Shilov, Morgan's, Hardy's, and Cowling-Price in the setting of this paper.

The organization of this paper is as follows: In Section 2 we recapitulate some definitions and basic notations, as well as some results from literature. In Section 3 we study the main properties of the eigenfunction $\Psi_{A, \varepsilon}$. In particular, we obtain estimates for the growth of $\Psi_{A, \varepsilon}$ and of its partial derivatives. In Section 4 we derive a Laplace type representation of the eigenfunction $\Psi_{A, \varepsilon}$. Next, we prove the existence and the positivity of the intertwining operator $V_{A, \varepsilon}$ between $\Lambda_{A, \varepsilon}$ and the ordinary derivative.

\section{BACKGROUND}

In this introductory section we present results from [5, 6, 32, 33].

Throughout this paper we will denote by $A$ a function on $\mathbb{R}$ satisfying the following hypotheses: 
(H1) $A(x)=|x|^{2 \alpha+1} B(x)$, where $\alpha>-\frac{1}{2}$ and $B$ is any even, positive and smooth function on $\mathbb{R}$ with $B(0)=1$.

(H2) $A$ is increasing and unbounded on $\mathbb{R}_{+}$.

(H3) $A^{\prime} / A$ is a decreasing and smooth function on $\mathbb{R}_{+}^{*}$, and hence the limit $2 \varrho:=\lim _{x \rightarrow+\infty} A^{\prime}(x) / A(x) \geq$ 0 exists.

(H4) There exists a constant $\delta>0$ such that for all $x \in\left[x_{0}, \infty\right)$ (for some $x_{0}>0$ ),

$$
\frac{A^{\prime}(x)}{A(x)}= \begin{cases}2 \varrho+e^{-\delta x} D(x) & \text { if } \varrho>0, \\ \frac{2 \alpha+1}{x}+e^{-\delta x} D(x) & \text { if } \varrho=0,\end{cases}
$$

with $D$ being a smooth function bounded together with its derivatives.

Such a function $A$ is called a Chébli function. From (H1) it follows that

$$
\frac{A^{\prime}(x)}{A(x)}=\frac{2 \alpha+1}{x}+C(x), \quad x \neq 0,
$$

where $C:=B^{\prime} / B$ is an odd and smooth function on $\mathbb{R}$.

Let $\Delta_{A}$, or simply $\Delta$, be the following second order differential operator

$$
\Delta=\frac{d^{2}}{d x^{2}}+\frac{A^{\prime}(x)}{A(x)} \frac{d}{d x}
$$

For $\mu \in \mathbb{C}$, we consider the Cauchy problem

$$
\Delta f(x)=-\left(\mu^{2}+\varrho^{2}\right) f(x) \text { with } f(0)=1 \text { and } f^{\prime}(0)=0 .
$$

In [6] the author proved that the system (2.3) admits a unique solution $\varphi_{\mu}$. For every $\mu \in \mathbb{C}$, the solution $\varphi_{\mu}$ is an even smooth function on $\mathbb{R}$ and the map $\mu \mapsto \varphi_{\mu}(x)$ is analytic. The following Laplace type representation of $\varphi_{\mu}$ can be found in [6] (see also [32]).

Lemma 2.1. For every $x \in \mathbb{R}^{*}$ there exists a probability measure $v_{x}$ on $\mathbb{R}$ supported in $[-|x|,|x|]$ such that for all $\mu \in \mathbb{C}$

$$
\varphi_{\mu}(x)=\int_{-|x|}^{|x|} e^{(i \mu-\varrho) t} v_{x}(d t)
$$

Also, for $x \in \mathbb{R}^{*}$, there is a non-negative even continuous function $K(|x|, \cdot)$ supported in $[-|x|,|x|]$ such that for all $\mu \in \mathbb{C}$

$$
\varphi_{\mu}(x)=\int_{0}^{|x|} K(|x|, t) \cos (\mu t) d t
$$

The following estimates of the eigenfunctions $\varphi_{\mu}$ can be found in [5, 6, 33].

Lemma 2.2. Let $\mu \in \mathbb{C}$ such that $|\operatorname{Im} \mu| \leq \varrho$. Then

1) $\varphi_{ \pm i o}(x)=1, \varphi_{-\mu}(x)=\varphi_{\mu}(x)$ and $\left|\varphi_{\mu}(x)\right| \leq 1$.

2) $e^{-\varrho|x|} \leq \varphi_{0}(x) \leq c(|x|+1) e^{-\varrho|x|}$.

3) $\left|\varphi_{\mu}(x)\right| \leq \varphi_{i \operatorname{Im} \mu}(x) \leq e^{|\operatorname{Im} \mu \|| x \mid} \varphi_{0}(x)$.

4) $\left|\varphi_{\mu}^{\prime}(x)\right| \leq c\left(\varrho^{2}+|\mu|^{2}\right) e^{|\operatorname{Im} \mu \| x|} \varphi_{0}(x)$.

We close this section by giving some basic results of (the analogue of) the Abel transform associated to the second order differential operator $\Delta$. 
Denote by $\mathscr{D}_{e}(\mathbb{R})$ the space of even and compactly supported functions in $C^{\infty}(\mathbb{R})$. In [32] the author has proved that the Abel transform defined on $\mathscr{D}_{e}(\mathbb{R})$ by

$$
\mathscr{A} f(y)=\frac{1}{2} \int_{|x|>|y|} K(|x|, y) f(x) A(x) d x
$$

is an automorphism of $\mathscr{D}_{e}(\mathbb{R})$ satisfying

$$
\mathscr{A} \circ\left(\Delta+\varrho^{2}\right)=\frac{d^{2}}{d x^{2}} \circ \mathscr{A} .
$$

\section{A FAMILY OF DIFFERENTIAL-REFLECTION OPERATORS}

For $\varepsilon \in \mathbb{R}$ we consider the following one-dimensional differential-reflection operators

$$
\Lambda_{A, \varepsilon} f(x)=f^{\prime}(x)+\frac{A^{\prime}(x)}{A(x)}\left(\frac{f(x)-f(-x)}{2}\right)-\varepsilon \varrho f(-x) .
$$

In view of $(2.1)$ and the hypothesis $(\mathrm{H} 4)$ on $A^{\prime} / A$, the space $\mathscr{D}(\mathbb{R})$ (of smooth functions with compact support on $\mathbb{R}$ ) and the space $\mathscr{S}(\mathbb{R})$ (of Schwartz functions on $\mathbb{R}$ ) are invariant under the action of $\Lambda_{A, \varepsilon}$.

Let $S$ denote the symmetry $(S f)(x):=f(-x)$. The following lemma is needed later. The easy proof is left to the reader.

Lemma 3.1. Let $f \in C^{\infty}(\mathbb{R})$ such that $\sup _{x \in \mathbb{R}}(1+|x|)^{r} e^{s|x|}\left|f^{(t)}(x)\right|<\infty$ for every $r, t \in \mathbb{N}$ and for some $2 \varrho \leq s<\infty$, and let $g \in C^{\infty}(\mathbb{R})$ such that $g$ and all its derivatives are at most of polynomial growth. Then

$$
\int_{\mathbb{R}} \Lambda_{A, \varepsilon} f(x) g(x) A(x) d x=-\int_{\mathbb{R}} f(x)\left(\Lambda_{A, \varepsilon}+2 \varepsilon \varrho S\right) g(x) A(x) d x .
$$

Let $\lambda \in \mathbb{C}$ and consider the initial data problem

$$
\Lambda_{A, \varepsilon} f(x)=i \lambda f(x) \quad \text { with } \quad f(0)=1,
$$

where $f: \mathbb{R} \rightarrow \mathbb{C}$. We have the following statement.

Theorem 3.2. Let $\lambda \in \mathbb{C}$. There exists a unique solution $\Psi_{A, \varepsilon}(\lambda, \cdot)$ to the problem (3.2). Further, for every $x \in \mathbb{R}$, the function $\lambda \mapsto \Psi_{A, \varepsilon}(\lambda, x)$ is analytic on $\mathbb{C}$. More explicitly:

(i) For $i \lambda \neq \varepsilon \varrho$,

$$
\Psi_{A, \varepsilon}(\lambda, x)=\varphi_{\mu_{\varepsilon}}(x)+\frac{1}{i \lambda-\varepsilon \varrho} \varphi_{\mu_{\varepsilon}}^{\prime}(x) \text { with } \mu_{\varepsilon}^{2}:=\lambda^{2}+\left(\varepsilon^{2}-1\right) \varrho^{2} .
$$

We may rewrite the solution (3.3) as

$$
\Psi_{A, \varepsilon}(\lambda, x)=\varphi_{\mu_{\varepsilon}}(x)+(i \lambda+\varepsilon \varrho) \frac{\operatorname{sgn}(x)}{A(x)} \int_{0}^{|x|} \varphi_{\mu_{\varepsilon}}(t) A(t) d t .
$$

(ii) For $i \lambda=\varepsilon \varrho$,

$$
\Psi_{A, \varepsilon}(\lambda, x)=1+2 \varepsilon \varrho \frac{\operatorname{sgn}(x)}{A(x)} \int_{0}^{|x|} A(t) d t .
$$

Proof. The proof is easy and standard.

The following positivity result is the basic ingredient in obtaining suitable growth estimates of $\Psi_{A, \varepsilon}$. 
Theorem 3.3. Assume that $-1 \leq \varepsilon \leq 1$. For all $\lambda \in i \mathbb{R}$, the function $\Psi_{A, \varepsilon}(\lambda, \cdot)$ is real and strictly positive.

Proof. The proof is inspired by [30]. If we take complex conjugates in (3.2), we see that $\overline{\Psi_{A, \varepsilon}(\lambda, \cdot)}$ and $\Psi_{A, \varepsilon}(\lambda, \cdot)$ satisfy the same system (3.2). Since $\overline{\Psi_{A, \varepsilon}(\lambda, 0)}=1$, the uniqueness part in Theorem 3.2 shows that $\overline{\Psi_{A, \varepsilon}(\lambda, x)}=\Psi_{A, \varepsilon}(\lambda, x)$ for all $x \in \mathbb{R}$.

Assume that $\Psi_{A, \varepsilon}(\lambda, \cdot)$ is not strictly positive. Since $\Psi_{A, \varepsilon}(\lambda, 0)=1>0$, it follows that $\Psi_{A, \varepsilon}(\lambda, \cdot)$ vanishes. Let $x_{0}$ be a zero of $\Psi_{A, \varepsilon}(\lambda, \cdot)$ so that

$$
\left|x_{0}\right|=\inf \left\{|x|: \Psi_{A, \varepsilon}(\lambda, x)=0\right\} .
$$

Since $\Psi_{A, \varepsilon}(\lambda, 0)=1$ we have $\Psi_{A, \varepsilon}(\lambda, x) \geq 0$ on $\left[-\left|x_{0}\right|,\left|x_{0}\right|\right]$. In particular $\Psi_{A, \varepsilon}\left(\lambda,-x_{0}\right) \geq 0$. We claim that

$$
\Psi_{A, \varepsilon}^{\prime}\left(\lambda, x_{0}\right)=0 \text { and } \Psi_{A, \varepsilon}\left(\lambda,-x_{0}\right)=0 .
$$

To prove (3.6), let us first assume that $x_{0}>0$. Then $\Psi_{A, \varepsilon}^{\prime}\left(\lambda, x_{0}\right) \leq 0$. Moreover,

$$
\Psi_{A, \varepsilon}^{\prime}(\lambda, x)=\left(\frac{A^{\prime}(x)}{2 A(x)}+\varepsilon \varrho\right)\left(\Psi_{A, \varepsilon}(\lambda,-x)-\Psi_{A, \varepsilon}(\lambda, x)\right)+(i \lambda+\varepsilon \varrho) \Psi_{A, \varepsilon}(\lambda, x) .
$$

Thus

$$
\Psi_{A, \varepsilon}^{\prime}\left(\lambda, x_{0}\right)=\left(\frac{A^{\prime}\left(x_{0}\right)}{2 A\left(x_{0}\right)}+\varepsilon \varrho\right) \Psi_{A, \varepsilon}\left(\lambda,-x_{0}\right) .
$$

From (3.8) it follows that $\Psi_{A, \varepsilon}^{\prime}\left(\lambda, x_{0}\right)$ is positive. This is due to the fact that $\varepsilon \geq-1$ and the fact that $A^{\prime} /(2 A)$ is a decreasing function on $\mathbb{R}_{+}^{*}$ and $\lim _{x \rightarrow+\infty} A^{\prime}(x) / 2 A(x)=\varrho$. We deduce that

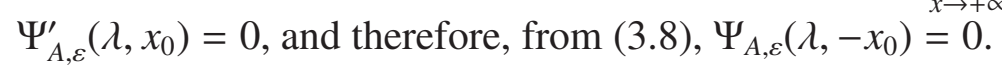

Now, let us assume that $x_{0}<0$. Then $\Psi_{A, \varepsilon}^{\prime}\left(\lambda, x_{0}\right) \geq 0$. Moreover, for $x_{0}<0$, equation (3.8) implies $\Psi_{A, \varepsilon}^{\prime}\left(\lambda, x_{0}\right) \leq 0$. This is due to $\varepsilon \leq 1$ and to assumptions on $A^{\prime} /(2 A)$. Then, as above, we conclude that $\Psi_{A, \varepsilon}^{\prime}\left(\lambda, x_{0}\right)=0$, and once again appealing to (3.8) we have $\Psi_{A, \varepsilon}\left(\lambda,-x_{0}\right)=0$. This finishes the proof of the claim (3.6).

Starting this time from $\Psi_{A, \varepsilon}\left(\lambda,-x_{0}\right)=0$ and proceeding analogously as in the case $\Psi_{A, \varepsilon}\left(\lambda, x_{0}\right)=$ 0 , we conclude that $\Psi_{A, \varepsilon}^{\prime}\left(\lambda,-x_{0}\right)=0$ and $\Psi_{A, \varepsilon}\left(\lambda, x_{0}\right)=0$.

In summary, $\Psi_{A, \varepsilon}\left(\lambda, \pm x_{0}\right)=0$ and $\Psi_{A, \varepsilon}^{\prime}\left(\lambda, \pm x_{0}\right)=0$. Differentiating (3.7), we see that the second derivative of $\Psi_{A, \varepsilon}(\lambda, \cdot)$ vanishes at $\pm x_{0}$. Repeating the same argument over and over again to get $\Psi_{A, \varepsilon}^{(k)}\left(\lambda, \pm x_{0}\right)=0$ for all $k \in \mathbb{N}$. Since $\Psi_{A, \varepsilon}(\lambda, \cdot)$ is a real analytic function, we deduce that $\Psi_{A, \varepsilon}(\lambda, x)=0$ for all $x \in \mathbb{R}$. This contradicts $\Psi_{A, \varepsilon}(\lambda, 0)=1$. Thus, either $\Psi_{A, \varepsilon}(\lambda, x)$ is strictly positive for all $x$, or it is strictly negative for all $x$. But since $\Psi_{A, \varepsilon}(\lambda, 0)=1$, it must be $\Psi_{A, \varepsilon}(\lambda, x)>0$ for all $x \in \mathbb{R}$.

The following theorem contains estimates for the growth of the eigenfunction $\Psi_{A, \varepsilon}$.

Theorem 3.4. Suppose that $-1 \leq \varepsilon \leq 1$ and $x \in \mathbb{R}$. Then:

1) For $\lambda \in \mathbb{R}$ we have $\left|\Psi_{A, \varepsilon}(\lambda, x)\right| \leq \sqrt{2}$.

2) For $\lambda=a+i b \in \mathbb{C}$ we have $\left|\Psi_{A, \varepsilon}(\lambda, x)\right| \leq \Psi_{A, \varepsilon}(i b, x)$.

3) For $\lambda=i b \in i \mathbb{R}$ we have $\Psi_{A, \varepsilon}(i b, x) \leq \Psi_{A, \varepsilon}(0, x) e^{|b||x|}$.

4) For $\lambda=0$ we distinguish the following two cases:

a) For $\varepsilon=0$, we have $\Psi_{A, 0}(0, x)=1$.

b) For $\varepsilon \neq 0$, there is a constant $c_{\varepsilon}>0$ such that $\Psi_{A, \varepsilon}(0, x) \leq c_{\varepsilon}(|x|+1) e^{-\varrho\left(1-\sqrt{1-\varepsilon^{2}}\right)|x|}$. 
Proof. 1) Assume that $\lambda \in \mathbb{R}$. Since $\Psi_{A, \varepsilon}(\lambda, x)$ is a solution of the problem (3.2), we deduce that

$$
\left\{\Psi_{A, \varepsilon}(\lambda, \pm x)\right\}^{\prime}=-\frac{A^{\prime}(x)}{2 A(x)}\left(\Psi_{A, \varepsilon}(\lambda, \pm x)-\Psi_{A, \varepsilon}(\lambda, \mp x)\right) \pm \varepsilon \varrho \Psi_{A, \varepsilon}(\lambda, \mp x) \pm i \lambda \Psi_{A, \varepsilon}(\lambda, \pm x) .
$$

By taking complex conjugates in (3.9), we obtain

$$
\begin{aligned}
& \left\{\left|\Psi_{A, \varepsilon}(\lambda, \pm x)\right|^{2}\right\}^{\prime}= \\
& =\operatorname{Re}\left\{-\frac{A^{\prime}(x)}{A(x)}\left(\Psi_{A, \varepsilon}(\lambda, \pm x)-\Psi_{A, \varepsilon}(\lambda, \mp x)\right) \overline{\Psi_{A, \varepsilon}(\lambda, \pm x)} \pm 2 \varepsilon \varrho \Psi_{A, \varepsilon}(\lambda, \mp x) \overline{\Psi_{A, \varepsilon}(\lambda, \pm x)}\right\} .
\end{aligned}
$$

Since $A^{\prime}(x) / A(x) \geq 2 \varrho \geq 0$ for all $x \in \mathbb{R}_{+}$, it follows that

$$
\left\{\left|\Psi_{A, \varepsilon}(\lambda,-x)\right|^{2}\right\}^{\prime}+\left\{\left|\Psi_{A, \varepsilon}(\lambda, x)\right|^{2}\right\}^{\prime}=-\frac{A^{\prime}(x)}{A(x)}\left|\Psi_{A, \varepsilon}(\lambda,-x)-\Psi_{A, \varepsilon}(\lambda, x)\right|^{2} \leq 0, \quad \forall x \in \mathbb{R}_{+} .
$$

This implies

$$
\left|\Psi_{A, \varepsilon}(\lambda,-x)\right|^{2}+\left|\Psi_{A, \varepsilon}(\lambda, x)\right|^{2} \leq\left|\Psi_{A, \varepsilon}(\lambda, 0)\right|^{2}+\left|\Psi_{A, \varepsilon}(\lambda, 0)\right|^{2}=2, \quad \forall x \in \mathbb{R}_{+} .
$$

As a consequence

$$
\left|\Psi_{A, \varepsilon}(\lambda,-x)\right| \leq \sqrt{2} \quad \text { and } \quad\left|\Psi_{A, \varepsilon}(\lambda, x)\right| \leq \sqrt{2}, \quad \forall x \in \mathbb{R}_{+} .
$$

2) For $\lambda=a+i b \in \mathbb{C}$ we define the function $Q_{\varepsilon, \lambda}(x):=\frac{\Psi_{A, \varepsilon}(\lambda, x)}{\Psi_{A, \varepsilon}(i b, x)}$. By Theorem 3.3 the function $Q_{\varepsilon, \lambda}$ is well defined. Using the identity (3.9) we have

$$
\begin{aligned}
\left\{\left|Q_{\varepsilon, \lambda}( \pm x)\right|^{2}\right\}^{\prime} & = \pm 2 \operatorname{Re}\left\{Q_{\varepsilon, \lambda}^{\prime}( \pm x) \overline{Q_{\varepsilon, \lambda}( \pm x)}\right\} \\
& =-2\left(\frac{A^{\prime}(x)}{2 A(x)} \pm \varepsilon \varrho\right)\left(\left|Q_{\varepsilon, \lambda}( \pm x)\right|^{2}-\operatorname{Re}\left\{Q_{\varepsilon, \lambda}(\mp x) \overline{Q_{\varepsilon, \lambda}( \pm x)}\right\}\right) \frac{\Psi_{A, \varepsilon}(i b, \mp x)}{\Psi_{A, \varepsilon}(i b, \pm x)} .
\end{aligned}
$$

Since $-1 \leq \varepsilon \leq 1$, then, by assumptions on the function $A^{\prime} /(2 A)$, we have

$$
\left(\frac{A^{\prime}(x)}{2 A(x)} \pm \varepsilon \varrho\right) \geq 0 \quad \forall x \in \mathbb{R}_{+} .
$$

It follows from (3.10) that for every $x \in \mathbb{R}_{+}$

$$
\left\{\left|Q_{\varepsilon, \lambda}( \pm x)\right|^{2}\right\}^{\prime} \leq-2\left(\frac{A^{\prime}(x)}{2 A(x)} \pm \varepsilon \varrho\right)\left|Q_{\varepsilon, \lambda}( \pm x)\right|\left(\left|Q_{\varepsilon, \lambda}( \pm x)\right|-\left|Q_{\varepsilon, \lambda}(\mp x)\right|\right) \frac{\Psi_{A, \varepsilon}(i b, \mp x)}{\Psi_{A, \varepsilon}(i b, \pm x)} .
$$

Thus we can conclude that

$$
\left\{\left|Q_{\varepsilon, \lambda}( \pm x)\right|^{2}\right\}^{\prime} \leq 0 \quad \text { if } \quad\left|Q_{\varepsilon, \lambda}( \pm x)\right| \geq\left|Q_{\varepsilon, \lambda}(\mp x)\right| .
$$

As a real analytic function of $x,\left|Q_{\varepsilon, \lambda}(x)\right|^{2}$ and $\left|Q_{\varepsilon, \lambda}(-x)\right|^{2}$ coincide either everywhere or on a discrete subset of $\mathbb{R}$ with no accumulation point. In the first case, $\left|Q_{\varepsilon, \lambda}(x)\right|^{2}=\left|Q_{\varepsilon, \lambda}(-x)\right|^{2}$ is a decreasing function of $x \in \mathbb{R}_{+}$. In the second case, for $x \in \mathbb{R}_{+}$, let

$$
M(x):=\max \left\{\left|Q_{\varepsilon, \lambda}(x)\right|^{2},\left|Q_{\varepsilon, \lambda}(-x)\right|^{2}\right\} .
$$

If $\left|Q_{\varepsilon, \lambda}(x)\right|>\left|Q_{\varepsilon, \lambda}(-x)\right|$, then $M(x)=\left|Q_{\varepsilon, \lambda}(x)\right|^{2}$ and $M^{\prime}(x)=\left\{\left|Q_{\varepsilon, \lambda}(x)\right|^{2}\right\}^{\prime}<0$. If $\left|Q_{\varepsilon, \lambda}(x)\right|<$ $\left|Q_{\varepsilon, \lambda}(-x)\right|$, then $M(x)=\left|Q_{\varepsilon, \lambda}(-x)\right|^{2}$ and $M^{\prime}(x)=\left\{\left|Q_{\varepsilon, \lambda}(-x)\right|^{2}\right\}^{\prime}<0$. If $\left|Q_{\varepsilon, \lambda}(x)\right|=\left|Q_{\varepsilon, \lambda}(-x)\right|$ for some $x \in \mathbb{R}_{+}$, then $M$ has left and right derivatives at $x$, which are non-positive. Thus $M$ is decreasing on $\mathbb{R}_{+}$. In conclusion, for every $x \in \mathbb{R}_{+},\left|Q_{\varepsilon, \lambda}(x)\right|^{2} \leq M(0)=1$ and $\left|Q_{\varepsilon, \lambda}(-x)\right|^{2} \leq$ $M(0)=1$. That is for every $x \in \mathbb{R}$, we have $\left|Q_{\varepsilon, \lambda}(x)\right| \leq\left|Q_{\varepsilon, \lambda}(0)\right|=1$. This finishes the proof of 
the second statement.

3) We proceed analogously to the function $Q_{\varepsilon, \lambda}$ above by considering the function

$$
R_{\varepsilon, b}(x):=\frac{\Psi_{A, \varepsilon}(i b, x) e^{-|b||x|}}{\Psi_{A, \varepsilon}(0, x)}
$$

4) The fact that $\Psi_{A, 0}(0, x)=1$ follows immediately from (3.5). Assume that $\varepsilon \neq 0$. In this case

$$
\Psi_{A, \varepsilon}(0, x)=\varphi_{\mu_{\varepsilon}^{0}}(x)-\frac{1}{\varepsilon \varrho} \varphi_{\mu_{\varepsilon}^{0}}^{\prime}(x),
$$

where $\mu_{\varepsilon}^{0}$ satisfies $\left(\mu_{\varepsilon}^{0}\right)^{2}=\left(\varepsilon^{2}-1\right) \varrho^{2}$. Since $|\varepsilon| \leq 1$, it follows from Lemma 2.2 2), 3), 4) that there exists a positive constant $c_{\varepsilon}$ such that $\Psi_{A, \varepsilon}(0, x) \leq c_{\varepsilon}(|x|+1) e^{-\varrho\left(1-\sqrt{1-\varepsilon^{2}}\right)|x|}$.

Henceforth, we will assume that $-1 \leq \varepsilon \leq 1$. We remind the reader that $\varphi_{i \varrho}(x)=1$ and $\varphi_{i \sqrt{1-\varepsilon^{2}} \varrho}(x) \leq c(|x|+1) e^{-\varrho\left(1-\sqrt{1-\varepsilon^{2}}\right)|x|} ;$ see Lemma 2.2 .

Theorem 3.5. 1) Assume that $\lambda \in \mathbb{C}$ and $|x| \geq x_{0}$ with $x_{0}>0$. Given $N \in \mathbb{N}$, there is a positive constant c such that

$$
\left|\frac{\partial^{N}}{\partial x^{N}} \Psi_{A, \varepsilon}(\lambda, x)\right| \leq c(|\lambda|+1)^{N} e^{|\operatorname{Im} \lambda||x|} \varphi_{i \sqrt{1-\varepsilon^{2}} \varrho}(x) .
$$

2) Assume that $\lambda \in \mathbb{C}$ and $x \in \mathbb{R}$. Given $M \in \mathbb{N}$, there is a positive constant $c$ such that

$$
\left|\frac{\partial^{M}}{\partial \lambda^{M}} \Psi_{A, \varepsilon}(\lambda, x)\right| \leq c|x|^{M} e^{|\operatorname{Im} \lambda||x|} \varphi_{i \sqrt{1-\varepsilon^{2}} \varrho}(x) .
$$

Proof. 1) If $N=0$ this is nothing but Theorem 3.42), 3) and 4). So assume $N \geq 1$. On the one hand, $\Psi_{A, \varepsilon}(\lambda, x)$ satisfies the following equation

$$
\Psi_{A, \varepsilon}^{\prime}(\lambda, x)=-\frac{A^{\prime}(x)}{2 A(x)}\left(\Psi_{A, \varepsilon}(\lambda, x)-\Psi_{A, \varepsilon}(\lambda,-x)\right)+\varepsilon \varrho \Psi_{A, \varepsilon}(\lambda,-x)+i \lambda \Psi_{A, \varepsilon}(\lambda, x) .
$$

This allows us to express the derivatives of $\Psi_{A, \varepsilon}(\lambda, \cdot)$ in terms of lower order derivatives. On the other hand, since $A^{\prime} /(2 A)$ satisfies the hypothesis (H4), it follows that there exists a positive constant $C$ such that

$$
\left|\left(\frac{A^{\prime}(x)}{2 A(x)}\right)^{(N)}\right| \leq C, \quad \forall|x| \geq x_{0} \text { with } x_{0}>0 .
$$

Now the estimate (3.11) can be proved by induction on $N$.

2) Recall that the mapping $\lambda \mapsto \Psi_{A, \varepsilon}(\lambda, x)$ is entire, for every $x \in \mathbb{R}$, and that

$$
\left|\Psi_{A, \varepsilon}(\lambda, x)\right| \leq c e^{|\operatorname{Im} \lambda||x|} \varphi_{i \sqrt{1-\varepsilon^{2}} \varrho}(x)
$$

for all $\lambda \in \mathbb{C}$ and $x \in \mathbb{R}$. If $M=0$ this is just (3.12). So assume $M>0$. If $x=0$, the statement follows from Liouville's theorem. If $x \neq 0$, apply Cauchy's integral formula for $\Psi_{A, \varepsilon}(\lambda, x)$ over a circle with radius proportional to $\frac{1}{|x|}$, centered at $\lambda$ in the complex plane. 


\section{AN INTERTWINING OPERATOR}

4.1. A Laplace type representation of the eigenfunctions. In this section we will show that $\Psi_{A, \varepsilon}(\lambda, \cdot)$ can be expressed as the Laplace transform of a compactly supported function. In the literature this is the so-called Mehler's type formula.

Denote by $C_{e}^{\infty}(\mathbb{R})$ the space of even functions in $C^{\infty}(\mathbb{R})$. For $f \in C_{e}^{\infty}(\mathbb{R})$ we set

$$
\mathscr{E}_{\varepsilon} f(x):=f(x)-\frac{\varrho_{\varepsilon}|x|}{2} \int_{|y|<|x|} f(y) \frac{J_{1}\left(\varrho_{\varepsilon} \sqrt{x^{2}-y^{2}}\right)}{\sqrt{x^{2}-y^{2}}} d y,
$$

where $J_{1}$ is the Bessel function of the first kind and $\varrho_{\varepsilon}:=\sqrt{1-\varepsilon^{2}} \varrho$. If $\varepsilon= \pm 1$, then $\varrho_{ \pm 1}=0$, and therefore $\mathscr{E}_{ \pm 1}=\mathrm{id}$. The following statement is nothing but a reformulation of Proposition 2.1 in [31]. See also Theorem 5.1 in [21].

Proposition 4.1. The integral transform $\mathscr{E}_{\varepsilon}$ is an automorphism of $C_{e}^{\infty}(\mathbb{R})$ satisfying

$$
\frac{d^{2}}{d x^{2}} \circ \mathscr{E}_{\varepsilon}=\mathscr{E}_{\varepsilon} \circ\left(\frac{d^{2}}{d x^{2}}-\varrho_{\varepsilon}^{2}\right) \quad \text { and } \quad \mathscr{E}_{\varepsilon} f(0)=f(0)
$$

The inverse transform $\mathscr{E}_{\varepsilon}^{-1}$ is given by

$$
\mathscr{E}_{\varepsilon}^{-1} f(x)=f(x)+\frac{\varrho_{\varepsilon}|x|}{2} \int_{|y|<|x|} f(y) \frac{I_{1}\left(\varrho_{\varepsilon} \sqrt{x^{2}-y^{2}}\right)}{\sqrt{x^{2}-y^{2}}} d y,
$$

where $I_{1}$ is the modified Bessel function of the first kind.

Let $\mathscr{D}_{e}(\mathbb{R})$ be the space of even functions in $\mathscr{D}(\mathbb{R})$. For $g \in \mathscr{D}_{e}(\mathbb{R})$ put

$$
{ }^{\mathrm{t}} \mathscr{E}_{\varepsilon} g(y)=g(y)-\frac{\varrho_{\varepsilon}}{2} \int_{|x|>|y|}|x| g(x) \frac{J_{1}\left(\varrho_{\varepsilon} \sqrt{x^{2}-y^{2}}\right)}{\sqrt{x^{2}-y^{2}}} d x .
$$

We may rewrite ${ }^{\mathrm{t}} \mathscr{E}_{\varepsilon} g$ as

$$
{ }^{\mathrm{t}} \mathscr{E}_{\varepsilon} g(y)=-\int_{|y|}^{\infty} g^{\prime}(x) J_{0}\left(\varrho_{\varepsilon} \sqrt{x^{2}-y^{2}}\right) d x
$$

Below we will show that $\mathscr{D}_{e}(\mathbb{R})$ is stable by ${ }^{\mathrm{t}} \mathscr{E}_{\varepsilon}$. Thus, one may check that for all $f \in C_{e}^{\infty}(\mathbb{R})$ and all $g \in \mathscr{D}_{e}(\mathbb{R})$,

$$
\int_{\mathbb{R}} \mathscr{E}_{\varepsilon} f(x) g(x) d x=\int_{\mathbb{R}} f(y){ }^{\mathrm{t}} \mathscr{E}_{\varepsilon} g(y) d y .
$$

Theorem 4.2. The transform integral ${ }^{\mathrm{t}} \mathscr{E}_{\varepsilon}$ is an automorphism of $\mathscr{D}_{e}(\mathbb{R})$ satisfying

$$
{ }^{\mathrm{t}} \mathscr{E}_{\varepsilon} \circ \frac{d^{2}}{d x^{2}}=\left(\frac{d^{2}}{d x^{2}}-\varrho_{\varepsilon}^{2}\right) \circ{ }^{\mathrm{t}} \mathscr{E}_{\varepsilon}
$$

The inverse transform ${ }^{\mathrm{t}} \mathscr{E}_{\varepsilon}^{-1}$ is given by

$$
{ }^{\mathrm{t}} \mathscr{\mathscr { O }}_{\varepsilon}^{-1} g(y)=g(y)+\frac{\varrho_{\varepsilon}}{2} \int_{|x|>|y|}|x| g(x) \frac{I_{1}\left(\varrho_{\varepsilon} \sqrt{x^{2}-y^{2}}\right)}{\sqrt{x^{2}-y^{2}}} d x=-\int_{|y|}^{\infty} g^{\prime}(x) I_{0}\left(\varrho_{\varepsilon} \sqrt{x^{2}-y^{2}}\right) d x .
$$

Proof. It is clear that ${ }^{\mathrm{t}} \mathscr{E}_{\varepsilon} g$ is an even function whenever $g$ is even. A direct calculation gives the intertwining property (4.3), which we may rewrite as $D^{2} \circ^{\mathrm{t}} \mathscr{E}_{\varepsilon}={ }^{\mathrm{t}} \mathscr{E}_{\varepsilon} \circ\left(D^{2}+\varrho_{\varepsilon}^{2}\right)$, where $D$ denotes the ordinary derivative. Thus, for all $N \in \mathbb{N}$ and for all $y \in \mathbb{R}_{+}$, we have

$$
D^{2 N} \circ{ }^{\mathrm{t}} \mathscr{E}_{\varepsilon} g(y)={ }^{\mathrm{t}} \mathscr{E}_{\varepsilon} \circ\left(D^{2}+\varrho_{\varepsilon}^{2}\right)^{N} g(y)=-\int_{8}^{\infty} J_{0}\left(\varrho_{\varepsilon} \sqrt{x^{2}-y^{2}}\right) D\left(D^{2}+\varrho_{\varepsilon}^{2}\right)^{N} g(x) d x .
$$


Using the well know fact that $\left|J_{0}(r)\right| \leq 1$ for all $r \in \mathbb{R}_{+}$, it follows that if $\operatorname{supp}(g) \subset[-a, a]$, then there exists a constant $c$ such that

$$
\sup _{y \in[-a, a]}\left|D^{2 N} \circ{ }^{\mathrm{t}} \mathscr{E}_{\varepsilon} g(y)\right| \leq c \sup _{x \in[-a, a]}\left|D^{M} g(x)\right|<\infty,
$$

for some positive integer $M$. Thus, the space $\mathscr{D}_{e}(\mathbb{R})$ is stable by ${ }^{\mathrm{t}} \mathscr{E}_{\varepsilon}$.

Recall that we may rewrite ${ }^{\mathrm{t}} \mathscr{E}_{\varepsilon}$ and its "potential" inverse transform respectively as

$$
{ }^{\mathrm{t}} \mathscr{E}_{\varepsilon} g(y)=-\int_{|y|}^{\infty} g^{\prime}(x) J_{0}\left(\varrho_{\varepsilon} \sqrt{x^{2}-y^{2}}\right) d x \quad \text { and } \quad{ }^{\mathrm{t}} \mathscr{E}_{\varepsilon}^{-1} g(y)=-\int_{|y|}^{\infty} g^{\prime}(x) I_{0}\left(\varrho_{\varepsilon} \sqrt{x^{2}-y^{2}}\right) d x
$$

We will assume that $y>0$. Then

$$
\begin{aligned}
{ }^{\mathrm{t}} \mathscr{E}_{\varepsilon}\left({ }^{\mathrm{t}} \mathscr{O}_{\varepsilon}^{-1} g\right)(y)= & \int_{x>y}\left\{\int_{s>x} g^{\prime}(s) I_{0}\left(\varrho_{\varepsilon} \sqrt{s^{2}-x^{2}}\right) d s\right\}^{\prime} J_{0}\left(\varrho_{\varepsilon} \sqrt{x^{2}-y^{2}}\right) d x \\
= & -\int_{x>y} g^{\prime}(x) J_{0}\left(\varrho_{\varepsilon} \sqrt{x^{2}-y^{2}}\right) d x \\
& +\int_{x>y}\left\{\int_{s>x} g^{\prime}(s) \partial_{x} I_{0}\left(\varrho_{\varepsilon} \sqrt{s^{2}-x^{2}}\right) d s\right\} J_{0}\left(\varrho_{\varepsilon} \sqrt{x^{2}-y^{2}}\right) d x
\end{aligned}
$$

Integration by parts implies

$$
\int_{s>x} g^{\prime}(s) \partial_{x} I_{0}\left(\varrho_{\varepsilon} \sqrt{s^{2}-x^{2}}\right) d s=\frac{\varrho_{\varepsilon}^{2}}{2} x g(x)-\int_{s>x} g(s) \partial_{s} \partial_{x} I_{0}\left(\varrho_{\varepsilon} \sqrt{s^{2}-x^{2}}\right) d s .
$$

Above we have used the fact that $I_{0}^{\prime}(z)=I_{1}(z)$ and that the function $\left(\frac{z}{2}\right)^{-v} I_{v}(z)$ is normalized at 0 by 1 . Thus,

$$
\begin{aligned}
{ }^{\mathrm{t}} \mathscr{E}_{\varepsilon}\left({ }^{\mathrm{t}} \mathscr{O}_{\varepsilon}^{-1} g\right)(y) & =\int_{x>y}\left(\frac{\varrho_{\varepsilon}^{2}}{2} x g(x)-g^{\prime}(x)\right) J_{0}\left(\varrho_{\varepsilon} \sqrt{x^{2}-y^{2}}\right) d x \\
& -\int_{s>y} g(s)\left\{\int_{y}^{s} J_{0}\left(\varrho_{\varepsilon} \sqrt{x^{2}-y^{2}}\right) \partial_{s} \partial_{x} I_{0}\left(\varrho_{\varepsilon} \sqrt{s^{2}-x^{2}}\right) d x\right\} d s .
\end{aligned}
$$

Next, we will compute the integral within brackets on the right hand side of the identity above. On the one hand, since $I_{0}^{\prime}(z)=I_{1}(z)$, we have

$$
\begin{aligned}
\partial_{s} \partial_{x} I_{0}\left(\varrho_{\varepsilon} \sqrt{s^{2}-x^{2}}\right) & =-\varrho_{\varepsilon} x \partial_{s}\left(\left(s^{2}-x^{2}\right)^{-1 / 2} I_{1}\left(\varrho_{\varepsilon} \sqrt{s^{2}-x^{2}}\right)\right) \\
& =\varrho_{\varepsilon} \frac{x s}{\left(s^{2}-x^{2}\right)^{3 / 2}} I_{1}\left(\varrho_{\varepsilon} \sqrt{s^{2}-x^{2}}\right)-\varrho_{\varepsilon}^{2} \frac{x s}{\left(s^{2}-x^{2}\right)} I_{1}^{\prime}\left(\varrho_{\varepsilon} \sqrt{s^{2}-x^{2}}\right) \\
& =-\varrho_{\varepsilon}^{2} \frac{x s}{\left(s^{2}-x^{2}\right)} I_{2}\left(\varrho_{\varepsilon} \sqrt{s^{2}-x^{2}}\right) .
\end{aligned}
$$

Above we have used the well known differentiation identity $I_{v}^{\prime}(z)=I_{v+1}(z)+\frac{v}{z} I_{v}(z)$. On the other hand, using the following integral formula (see [15, formula (1), page 725])

$$
\int_{0}^{a} x^{\mu+1}\left(a^{2}-x^{2}\right)^{-\mu / 2-1} J_{\mu}(x) I_{v}\left(\sqrt{a^{2}-x^{2}}\right) d x=\frac{\left(\frac{a}{2}\right)^{\mu} \Gamma\left(\frac{v-\mu}{2}\right)}{2 \Gamma\left(\frac{v+\mu}{2}+1\right)} J_{v}(a), \quad \operatorname{Re} v>\operatorname{Re} \mu>-1
$$


we have

$$
\begin{aligned}
\int_{y}^{s} J_{0}\left(\varrho_{\varepsilon} \sqrt{x^{2}-y^{2}}\right) \partial_{s} \partial_{x} I_{0}\left(\varrho_{\varepsilon} \sqrt{s^{2}-x^{2}}\right) d x & =-\frac{\varrho_{\varepsilon}^{2}}{2} s J_{2}\left(\varrho_{\varepsilon} \sqrt{s^{2}-y^{2}}\right) \\
& =-\varrho_{\varepsilon} \frac{s}{\sqrt{s^{2}-y^{2}}} J_{1}\left(\varrho_{\varepsilon} \sqrt{s^{2}-y^{2}}\right)+\frac{\varrho_{\varepsilon}^{2}}{2} s J_{0}\left(\varrho_{\varepsilon} \sqrt{s^{2}-y^{2}}\right) \\
& =\partial_{s}\left(J_{0}\left(\varrho_{\varepsilon} \sqrt{s^{2}-y^{2}}\right)\right)+\frac{\varrho_{\varepsilon}^{2}}{2} s J_{0}\left(\varrho_{\varepsilon} \sqrt{s^{2}-y^{2}}\right) .
\end{aligned}
$$

Above we have used the recurrence relation $J_{v+1}(z)+J_{v-1}(z)=\frac{2 v}{z} J_{v}(z)$ and the fact that $J_{0}^{\prime}(z)=$ $-J_{1}(z)$. Consequently, ${ }^{\mathrm{t}} \mathscr{E}_{\varepsilon}\left({ }^{\mathrm{t}} \mathscr{E}_{\varepsilon}^{-1} g\right)(y)=g(y)$. Similarly one proves that ${ }^{\mathrm{t}} \mathscr{E}_{\varepsilon}^{-1}\left({ }^{\mathrm{t}} \mathscr{E}_{\varepsilon} g\right)=g$.

Recall from Lemma 2.1 the Laplace representation of $\varphi_{\mu}$. The following alternative Laplace representation of $\varphi_{\mu}$ is needed for later use. For $x \in \mathbb{R}$ and $y \in \mathbb{R}_{+}$put

$$
K_{\varepsilon}(|x|, y):={ }^{\mathrm{t}} \mathscr{E}_{\varepsilon}^{-1} K(|x|, \cdot)(y),
$$

where $K(|x|, \cdot)$ is as in (2.4). Observe that $K_{\varepsilon}(x, \cdot)$ is even, continuous and supported in [-|x|,|x|]. We note that if $\varepsilon= \pm 1$, then the transformation $\mathscr{E}_{ \pm 1}=\mathrm{id}$, and therefore $K_{ \pm 1}(x, y)=K(|x|, y)$.

Lemma 4.3. Let $\lambda \in \mathbb{C}$. The integral representation (2.4) can be rewritten as

$$
\varphi_{\mu_{\varepsilon}}(x)=\int_{0}^{|x|} K_{\varepsilon}(x, y) \cos (\lambda y) d y
$$

where the relationship between $\mu_{\varepsilon}$ and $\lambda$ is $\mu_{\varepsilon}^{2}=\lambda^{2}+\left(\varepsilon^{2}-1\right) \varrho^{2}$.

Proof. The cases $\varepsilon= \pm 1$ are trivial. So assume $\varepsilon \neq \pm 1$. Observe that we may rewrite $\mathscr{E}_{\varepsilon}$ as

$$
\mathscr{E}_{\varepsilon}(f)(y)=\left(y \int_{0}^{1} f(y t) J_{0}\left(\varrho_{\varepsilon} y \sqrt{1-t^{2}}\right) d t\right)_{y}^{\prime}
$$

for all $y>0$. Using the following integral formula (see [15, formula (7), page 722])

$$
\int_{0}^{a} \cos (c t) J_{0}\left(b \sqrt{a^{2}-t^{2}}\right) d t=\frac{\sin \left(a \sqrt{b^{2}+c^{2}}\right)}{\sqrt{b^{2}+c^{2}}}, \quad b>0
$$

we obtain

$$
\mathscr{E}_{\varepsilon}\left(\cos \left(\mu_{\varepsilon} \cdot\right)\right)(y)=\left(y \int_{0}^{1} \cos \left(\mu_{\varepsilon} y t\right) J_{0}\left(\varrho_{\varepsilon} y \sqrt{1-t^{2}}\right) d t\right)_{y}^{\prime}=\cos \left(\{\underbrace{\left(1-\varepsilon^{2}\right) \varrho^{2}+\mu_{\varepsilon}^{2}}_{=\lambda^{2}}\}^{1 / 2} y\right) .
$$

Thus $\mathscr{O}_{\varepsilon}^{-1}(\cos (\lambda \cdot))(y)=\cos \left(\mu_{\varepsilon} y\right)$. Now, the statement follows directly from the Laplace representation (2.4) of $\varphi_{\mu_{\varepsilon}}(x)$.

We now establish a Laplace type representation of the eigenfunction $\Psi_{A, \varepsilon}(\lambda, \cdot)$. Henceforth we will use the following notation

$$
G_{\varepsilon}(x, y):=\int_{|y|}^{|x|} K_{\varepsilon}(t, y) A(t) d t
$$

where $K_{\varepsilon}(t, y)$ is as in (4.4). The function $G_{\varepsilon}(x, \cdot)$ is even, continuous on its support [-|x|,|x|] and of class $C^{1}$ on ] - |x|,|x|[ (see e.g. [22, Lemma 2.8]). The following statement follows directly from Lemma 4.3 . 
Corollary 4.4. For all $\lambda \in \mathbb{C}$ the function $\Psi_{A, \varepsilon}(\lambda, \cdot): \mathbb{R}^{*} \rightarrow \mathbb{C}$ is the Laplace transform of a compactly supported function. More precisely,

$$
\Psi_{A, \varepsilon}(\lambda, x)=\int_{|y|<|x|} \mathbb{K}_{\varepsilon}(x, y) e^{i \lambda y} d y, \quad \forall x \in \mathbb{R}^{*},
$$

where

$$
\mathbb{K}_{\varepsilon}(x, y):=\frac{K_{\varepsilon}(x, y)}{2}+\varepsilon \varrho \frac{\operatorname{sgn}(x)}{2 A(x)} G_{\varepsilon}(x, y)-\frac{\operatorname{sgn}(x)}{2 A(x)} \partial_{y} G_{\varepsilon}(x, y) .
$$

4.2. The existence of an intertwining operator. This section is concerned with the existence of an intertwining operator between $\Lambda_{A, \varepsilon}$ and the ordinary derivative $d / d x$.

Recall from Section 2 the definition of the Abel transform $\mathscr{A}$ which is an automorphism of $\mathscr{D}_{e}(\mathbb{R})$. It is natural to define for smooth even functions the dual transform ${ }^{\mathrm{t}} \mathscr{A}$ of $\mathscr{A}$ in the following sense $\int_{\mathbb{R}} f(y) \mathscr{A} g(y) d y=\int_{\mathbb{R}}^{\mathrm{t}} \mathscr{A} f(x) g(x) A(x) d x$. In [32] the author showed that

$$
{ }^{\mathrm{t}} \mathscr{A} f(x)=\frac{1}{2} \int_{|u|<|x|} K(|x|, u) f(u) d u .
$$

where $K(|x|, u)$ is as in (2.4). Further, by [32, Theorem 5.1], the transform ${ }^{\mathrm{t}} \mathscr{A}$ is an automorphism of $C_{e}^{\infty}(\mathbb{R})$ (the space of even and smooth functions on $\mathbb{R}$ ) satisfying

$$
\left(\Delta+\varrho^{2}\right) \circ{ }^{\mathrm{t}} \mathscr{A}={ }^{\mathrm{t}} \mathscr{A} \circ \frac{d^{2}}{d x^{2}},
$$

where $\Delta$ is the operator (2.2).

For $-1 \leq \varepsilon \leq 1$ we define the integral transform $\mathscr{A}_{\varepsilon}$ on $\mathscr{D}_{e}(\mathbb{R})$ by

$$
\mathscr{A}_{\varepsilon} g(y)=\frac{1}{2} \int_{|x|>|y|} K_{\varepsilon}(x, y) g(x) A(x) d x,
$$

where the kernel $K_{\varepsilon}$ is as in (4.4). We note that for $\varepsilon= \pm 1$ the transform $\mathscr{A}_{\varepsilon}$ reduces to the Abel transform $\mathscr{A}$. We may think of $\mathscr{A}_{\varepsilon}$ as a deformation of the transform $\mathscr{A}$. Let ${ }^{\mathrm{t}} \mathscr{A}_{\varepsilon}$ be the linear mapping of $C_{e}^{\infty}(\mathbb{R})$ so that $\int_{\mathbb{R}} f(y) \mathscr{A}_{\varepsilon} g(y) d y=\int_{\mathbb{R}} \mathscr{A}_{\varepsilon} f(x) g(x) A(x) d x$ for $f \in C_{e}^{\infty}(\mathbb{R})$ and $g \in \mathscr{D}_{e}(\mathbb{R})$. Then

$$
{ }^{\mathrm{t}} \mathscr{A}_{\varepsilon} f(x)=\frac{1}{2} \int_{|y|<|x|} K_{\varepsilon}(x, y) f(y) d y .
$$

Notice that for $f \in C_{e}^{\infty}(\mathbb{R})$ and $g \in \mathscr{D}_{e}(\mathbb{R})$, the functions ${ }^{\mathrm{t}} \mathscr{A}_{\varepsilon} f$ and $\mathscr{A}_{\varepsilon} g$ belong respectively to $C_{e}^{\infty}(\mathbb{R})$ and $\mathscr{D}_{e}(\mathbb{R})$. Moreover,

$$
\mathscr{A}_{\varepsilon}={ }^{\mathrm{t}} \mathscr{E}_{\varepsilon}^{-1} \circ \mathscr{A} \text { and }{ }^{\mathrm{t}} \mathscr{A}_{\varepsilon}={ }^{\mathrm{t}} \mathscr{A} \circ \mathscr{E}_{\varepsilon}^{-1}
$$

The next corollary contains some additional properties of $\mathscr{A}_{\varepsilon}$ and ${ }^{\mathrm{t}} \mathscr{A}_{\varepsilon}$.

Corollary 4.5. Let $D$ be the ordinary derivative and let $\Delta$ be the operator (2.2). Then for all $\varepsilon \in \mathbb{R}$ we have:

1) $\mathscr{A}_{\varepsilon} \circ\left(\Delta+\varrho^{2}\right)=\left(D^{2}+\varrho_{\varepsilon}^{2}\right) \circ \mathscr{A}_{\varepsilon}$, where $\varrho_{\varepsilon}^{2}=\left(1-\varepsilon^{2}\right) \varrho^{2}$.

2) $\left(\Delta+\varrho^{2}\right) \circ{ }^{\mathrm{t}} \mathscr{A}_{\varepsilon}={ }^{\mathrm{t}} \mathscr{A}_{\varepsilon} \circ\left(D^{2}+\varrho_{\varepsilon}^{2}\right)$.

Proof. The first statement is an immediate consequence of (2.6) and (4.3). The second transmutation property follows from (4.2) and (4.6). 
For $f \in C^{\infty}(\mathbb{R})$ we define $V_{A, \varepsilon} f$ by

$$
V_{A, \varepsilon} f(x)=\int_{|y|<|x|} \mathbb{K}_{\varepsilon}(x, y) f(y) d y \quad \text { for } x \neq 0, \quad \text { and } \quad V_{A, \varepsilon} f(0)=f(0),
$$

where the kernel $\mathbb{K}_{\varepsilon}(x, y)$ is as in (4.5). Observe that

$$
\Psi_{A, \varepsilon}(\lambda, x)=V_{A, \varepsilon}\left(e^{i \lambda \cdot}\right)(x) .
$$

Lemma 4.6. The operator $V_{A, \varepsilon}$ can be expressed as

$$
V_{A, \varepsilon} f(x)=(\operatorname{id}+\varepsilon \varrho \mathscr{M})^{\mathrm{t}} \mathscr{A}_{\varepsilon} f_{e}(x)+\left(\varepsilon^{2} \varrho^{2} \mathscr{M}+\frac{d}{d x}\right)^{\mathrm{t}} \mathscr{A}_{\varepsilon}\left(I f_{o}\right)(x),
$$

where

$$
\mathscr{M} h(x):=\frac{\operatorname{sgn}(x)}{A(x)} \int_{0}^{|x|} h(t) A(t) d t \quad \text { and } \quad I h(x):=\int_{0}^{x} h(t) d t .
$$

Proof. As usual, we write $f$ as the superposition $f=f_{e}+f_{o}$ of an even function $f_{e}$ and an odd function $f_{o}$. On the one hand, we have

$$
\begin{aligned}
V_{A, \varepsilon} f_{e}(x) & =\int_{-|x|}^{|x|} \frac{K_{\varepsilon}(x, y)}{2} f_{e}(y) d y+\varepsilon \varrho \frac{\operatorname{sgn}(x)}{2 A(x)} \int_{-|x|}^{|x|} G_{\varepsilon}(x, y) f_{e}(y) d y \\
& ={ }^{\mathrm{t}} \mathscr{A}_{\varepsilon} f_{e}(x)+\varepsilon \varrho \mathscr{M} \circ{ }^{\mathrm{t}} \mathscr{A}_{\varepsilon} f_{e}(x) .
\end{aligned}
$$

On the other hand, $V_{A, \varepsilon} f_{o}(x)=-\frac{\operatorname{sgn}(x)}{A(x)} \int_{0}^{|x|} f_{o}(y) \partial_{y} G_{\varepsilon}(x, y) d y$. We claim that

$$
-\frac{\operatorname{sgn}(x)}{A(x)} \int_{0}^{|x|} f_{o}(y) \partial_{y} G_{\varepsilon}(x, y) d y=\left(\varepsilon^{2} \varrho^{2} \mathscr{M}+\frac{d}{d x}\right)^{\mathrm{t}} \mathscr{A}_{\varepsilon}\left(I f_{o}\right)(x),
$$

where $I f_{o}$ is as in (4.10). Indeed, let us first recall the following formula (easy to check)

$$
g^{\prime}(x)=\frac{\operatorname{sgn}(x)}{A(x)} \int_{0}^{|x|} \Delta g(t) A(t) d t
$$

for even functions. By invoking (4.12) in the first equality below and the transmutation property in Corollary 4.5.2 in the second equality below we have

$$
\begin{aligned}
\frac{d}{d x}{ }^{\mathrm{t}} \mathscr{A}_{\varepsilon}\left(I f_{o}\right)(x) & =\frac{\operatorname{sgn}(x)}{A(x)} \int_{0}^{|x|} \Delta^{\mathrm{t}} \mathscr{A}_{\varepsilon}\left(I f_{o}\right)(s) A(s) d s \\
& =\frac{\operatorname{sgn}(x)}{A(x)} \int_{0}^{|x|} \mathrm{t} \mathscr{A}_{\varepsilon}\left(\frac{d^{2}}{d x^{2}}-\varepsilon^{2} \varrho^{2}\right)\left(I f_{o}\right)(s) A(s) d s \\
& =\frac{\operatorname{sgn}(x)}{A(x)} \int_{0}^{|x|}\left\{\int_{0}^{s} K_{\varepsilon}(s, u) f_{o}^{\prime}(u) d u\right\} A(s) d s-\varepsilon^{2} \varrho^{2} \mathscr{M}^{\mathrm{t}} \mathscr{A}_{\varepsilon}\left(I f_{o}\right)(x) \\
& =\frac{\operatorname{sgn}(x)}{A(x)} \int_{0}^{|x|} f_{o}^{\prime}(u)\left\{\int_{u}^{|x|} K_{\varepsilon}(s, u) A(s) d s\right\} d u-\varepsilon^{2} \varrho^{2} \mathscr{M}^{\mathrm{t}}{ }^{\mathrm{t}} \mathscr{A}_{\varepsilon}\left(I f_{o}\right)(x) \\
& =-\frac{\operatorname{sgn}(x)}{A(x)} \int_{0}^{|x|} f_{o}(u) \partial_{u} G_{\varepsilon}(x, u) d u-\varepsilon^{2} \varrho^{2} \mathscr{M}^{\mathrm{t}} \mathscr{A}_{\varepsilon}\left(I f_{o}\right)(x) .
\end{aligned}
$$

This concludes the proof of claim (4.11), and therefore the proof of Lemma 4.6 . 
Theorem 4.7. The operator $V_{A, \varepsilon}$ is the unique automorphism of $C^{\infty}(\mathbb{R})$ such that

$$
\Lambda_{A, \varepsilon} \circ V_{A, \varepsilon}=V_{A, \varepsilon} \circ \frac{d}{d x}
$$

where $\Lambda_{A, \varepsilon}$ is the differential-reflection operator (3.1).

Proof. For the proof of this theorem it is more convenient to rewrite $V_{A, \varepsilon} f_{o}$ in (4.9) as

$$
V_{A, \varepsilon} f_{o}(x)=\mathscr{M} \circ{ }^{\mathrm{t}} \mathscr{A}_{\varepsilon}\left(f_{o}^{\prime}\right)(x) \text {. }
$$

Indeed,

$$
\begin{aligned}
V_{A, \varepsilon} f_{o}(x) & =\frac{d}{d x}{ }^{\mathrm{t}} \mathscr{A}_{\varepsilon}\left(I f_{o}\right)(x)+\varepsilon^{2} \varrho^{2} \mathscr{M}^{\mathrm{t}} \mathscr{A}_{\varepsilon}\left(I f_{o}\right)(x) \\
& =\mathscr{M} \Delta^{\mathrm{t}} \mathscr{A}_{\varepsilon}\left(I f_{o}\right)(x)+\varepsilon^{2} \varrho^{2} \mathscr{M}^{\mathrm{t}} \mathscr{A}_{\varepsilon}\left(I f_{o}\right)(x) \\
& =\mathscr{M}\left(\Delta+\varepsilon^{2} \varrho^{2}\right)^{\mathrm{t}} \mathscr{A}_{\varepsilon}\left(I f_{o}\right)(x) \\
& =\mathscr{M}^{\mathrm{t}} \mathscr{A}_{\varepsilon}\left(\left(I f_{o}\right)^{\prime \prime}\right)(x) \\
& =\mathscr{M}^{\mathrm{t}} \mathscr{\mathscr { A }}_{\varepsilon}\left(f_{o}^{\prime}\right)(x) .
\end{aligned}
$$

Let $C_{e}^{\infty}(\mathbb{R})$ and $C_{o}^{\infty}(\mathbb{R})$ be the subspaces of even and odd functions in $C^{\infty}(\mathbb{R})$, respectively. Firstly, the operator $d / d x$ is one to one from $C_{o}^{\infty}(\mathbb{R})$ onto $C_{e}^{\infty}(\mathbb{R})$, and $d / d x \circ I=I \circ d / d x=\mathrm{id}$. Secondly, the transform $\mathscr{M}$ is an isomorphism from $C_{e}^{\infty}(\mathbb{R})$ to $C_{o}^{\infty}(\mathbb{R})$ and its inverse is given by

$$
\mathscr{M}^{-1}=\frac{d}{d x}+\frac{A^{\prime}(x)}{A(x)} \mathrm{id} .
$$

Thus, from (4.9) and (4.13) it follows that $V_{A, \varepsilon}$ is an automorphism of $C^{\infty}(\mathbb{R})$. We now prove the transmutation property (4.7).

By (4.13) we have

$$
\Lambda_{A, \varepsilon}\left(V_{A, \varepsilon} f_{o}\right)=\Lambda_{A, \varepsilon}\left(\mathscr{M}^{\mathrm{t}} \mathscr{A}_{\varepsilon}\left(f_{o}^{\prime}\right)\right)=(\mathrm{id}+\varepsilon \varrho \mathscr{M})^{\mathrm{t}} \mathscr{A}_{\varepsilon}\left(f_{o}^{\prime}\right) .
$$

Above we have used the fact that $\Lambda_{A, \varepsilon} \circ \mathscr{M}=\mathrm{id}+\varepsilon \varrho \mathscr{M}$. Moreover, using (4.14), one can check that

$$
\begin{aligned}
\Lambda_{A, \varepsilon}\left(V_{A, \varepsilon} f_{e}\right) & =\Lambda_{A, \varepsilon}\left({ }^{\mathrm{t}} \mathscr{A}_{\varepsilon} f_{e}+\varepsilon \varrho \mathscr{M} \circ{ }^{\mathrm{t}} \mathscr{A}_{\varepsilon} f_{e}\right) \\
& =\frac{d}{d x}{ }^{\mathrm{t}} \mathscr{A}_{\varepsilon} f_{e}-\varepsilon \varrho^{\mathrm{t}} \mathscr{A}_{\varepsilon} f_{e}+\varepsilon \varrho\left(\frac{d}{d x}+\frac{A^{\prime}(x)}{A(x)}\right) \mathscr{M}^{\mathrm{t}} \mathscr{A}_{\varepsilon} f_{e}+\varepsilon^{2} \varrho^{2} \mathscr{M}^{\mathrm{t}} \mathscr{A}_{\varepsilon} f_{e} \\
& =\left(\frac{d}{d x}+\varepsilon^{2} \varrho^{2} \mathscr{M}\right){ }^{\mathrm{t}} \mathscr{A}_{\varepsilon} f_{e} .
\end{aligned}
$$

In summary,

$$
\Lambda_{A, \varepsilon}\left(V_{A, \varepsilon} f\right)=\left(\frac{d}{d x}+\varepsilon^{2} \varrho^{2} \mathscr{M}\right)^{\mathrm{t}} \mathscr{A}_{\varepsilon} f_{e}+(\mathrm{id}+\varepsilon \varrho \mathscr{M})^{\mathrm{t}} \mathscr{A}_{\varepsilon}\left(f_{o}^{\prime}\right) .
$$

Now, by invoking the expression (4.9) of the operator $V_{A, \varepsilon}$ we get

$$
V_{A, \varepsilon}\left(f_{e}^{\prime}\right)=\left(\varepsilon^{2} \varrho^{2} \mathscr{M}+\frac{d}{d x}\right)^{\mathrm{t}} \mathscr{A}_{\varepsilon} f_{e} \quad \text { and } \quad V_{A, \varepsilon}\left(f_{o}^{\prime}\right)=(\mathrm{id}+\varepsilon \varrho \mathscr{M})^{\mathrm{t}} \mathscr{A}_{\varepsilon}\left(f_{o}^{\prime}\right) .
$$

Therefore

$$
V_{A, \varepsilon}\left(f^{\prime}\right)=\left(\varepsilon^{2} \varrho^{2} \mathscr{M}+\frac{d}{d x}\right)^{\mathrm{t}} \mathscr{A}_{\varepsilon} f_{e}+(\mathrm{id}+\varepsilon \varrho \mathscr{M})^{\mathrm{t}} \mathscr{A}_{\varepsilon}\left(f_{o}^{\prime}\right) .
$$

This compares well with (4.15). The uniqueness of $V_{A, \varepsilon}$ is due to the fact that $\Psi_{A, \varepsilon}(\lambda, x)=$ $V_{A, \varepsilon}\left(e^{i \lambda \cdot}\right)(x)$ and that $V_{A, \varepsilon} f(0)=f(0)$. 
On the space $\mathscr{D}(\mathbb{R})$ of smooth functions with compact support, we consider the dual operator ${ }^{\mathrm{t}} V_{A, \varepsilon}$ of $V_{A, \varepsilon}$ in the sense that

$$
\int_{\mathbb{R}} V_{A, \varepsilon} f(x) g(x) A(x) d x=\int_{\mathbb{R}} f(y)^{\mathrm{t}} V_{A, \varepsilon} g(y) d y .
$$

This can be written as

$$
{ }^{\mathrm{t}} V_{A, \varepsilon} g(y)=\int_{|x|>|y|} \mathbb{K}_{\varepsilon}(x, y) g(x) A(x) d x .
$$

Lemma 4.8. The dual operator ${ }^{\mathrm{t}} V_{A, \varepsilon}$ can be expressed as

$$
{ }^{\mathrm{t}} V_{A, \varepsilon} g(y)=\mathscr{A}_{\varepsilon} g_{e}(y)-\left(\varepsilon \varrho-\frac{d}{d x}\right) \mathscr{A}_{\varepsilon}\left(J g_{o}\right)(y),
$$

where

$$
J h(x):=\int_{-\infty}^{x} h(t) d t
$$

Proof. The reader will have no trouble verifying that for every even function $f \in C^{\infty}(\mathbb{R})$ and every odd function $g \in \mathscr{D}(\mathbb{R})$

$$
\int_{\mathbb{R}} \mathscr{M} f(x) g(x) A(x) d x=-\int_{\mathbb{R}} f(x) \operatorname{Jg}(x) A(x) d x .
$$

Starting from the expression (4.9) of $V_{A, \varepsilon}$ in Lemma 4.6, and by invoking (4.13) in the first equality below, we obtain

$$
\begin{aligned}
& \int_{\mathbb{R}} V_{A, \varepsilon} f(x) g(x) A(x) d x \\
= & \int_{\mathbb{R}}\left\{{ }^{\mathrm{t}} \mathscr{A}_{\varepsilon} f_{e}(x) g_{e}(x)+\varepsilon \varrho \mathscr{M}^{\mathrm{t}} \mathscr{A}_{\varepsilon} f_{e}(x) g_{o}(x)+\mathscr{M}^{\mathrm{t}} \mathscr{A}_{\varepsilon} f_{o}^{\prime}(x) g_{o}(x)\right\} A(x) d x \\
= & \int_{\mathbb{R}}\left\{f_{e}(x) \mathscr{A}_{\varepsilon} g_{e}(x)-\varepsilon \varrho f_{e}(x) \mathscr{A}_{\varepsilon} J g_{o}(x)-f_{o}^{\prime}(x) \mathscr{A}_{\varepsilon} J g_{o}(x)\right\} d x \\
= & \int_{\mathbb{R}}\left\{f(x) \mathscr{A}_{\varepsilon} g_{e}(x)-\varepsilon \varrho f(x) \mathscr{A}_{\varepsilon} J g_{o}(x)+f_{o}(x) \frac{d}{d x} \mathscr{A}_{\varepsilon} J g_{o}(x)\right\} d x \\
= & \int_{\mathbb{R}} f(x)\left\{\mathscr{A}_{\varepsilon} g_{e}(x)-\varepsilon \varrho \mathscr{A}_{\varepsilon} J g_{o}(x)+\frac{d}{d x} \mathscr{A}_{\varepsilon} J g_{o}(x)\right\} d x .
\end{aligned}
$$

This finishes the proof of Lemma 4.8.

The operator ${ }^{\mathrm{t}} V_{A, \varepsilon}$ satisfies the following additional property.

Theorem 4.9. The operator ${ }^{\mathrm{t}} V_{A, \varepsilon}$ is the unique automorphism of $\mathscr{D}(\mathbb{R})$ satisfying the intertwining property

$$
\frac{d}{d x} \circ{ }^{\mathrm{t}} V_{A, \varepsilon}={ }^{\mathrm{t}} V_{A, \varepsilon} \circ\left(\Lambda_{A, \varepsilon}+2 \varepsilon \varrho S\right),
$$

where $S$ denotes the symmetry $(S f)(x):=f(-x)$.

Proof. The statement follows immediately from Lemma 3.1. 
4.3. The positivity of the intertwining operator. We shall say that a linear operator $L$ on $\mathscr{D}(\mathbb{R})$ is positive, if $L$ leaves the positive cone

$$
\mathscr{D}(\mathbb{R})_{+}:=\{f \in \mathscr{D}(\mathbb{R}): f(x) \geq 0 \text { for all } x \in \mathbb{R}\}
$$

invariant. The following statement is the central result of this section.

Theorem 4.10. For $-1 \leq \varepsilon \leq 1$, the intertwining operator $V_{A, \varepsilon}$ is positive.

For $\varepsilon=0$ and 1, Theorem 4.10 is known (cf. [36] and [37]), while for $A(x)=A_{\alpha}(x)=|x|^{2 \alpha+1}$ and $\varepsilon$ arbitrary (the Dunkl setting), Theorem 4.10 goes back to [27]. However, the case $-1 \leq$ $\varepsilon \leq 1$ has to overcome some technical difficulties compared to $\varepsilon=0$ and 1 , as $\varepsilon$ could be positive as well as negative.

The proof of the above theorem affords several steps, the crucial one being the positivity of $V_{A, \varepsilon}\left(p_{s}(u, \cdot)\right)(x)$ for every $s>0$ and $u, x \in \mathbb{R}$, where

$$
p_{s}(u, v):=\frac{e^{-\frac{(u-v)^{2}}{4 s}}}{2 \sqrt{\pi s}}
$$

denotes the Euclidean heat kernel.

For simplicity we will write $W_{\varepsilon}(s ; u, x)$ instead of $V_{A, \varepsilon}\left(p_{s}(u, \cdot)\right)(x)$. Below we list some properties of $W_{\varepsilon}(s ; u, x)$.

Lemma 4.11. For every $s>0$ and $u, x \in \mathbb{R}$, we have

1) $W_{\varepsilon}(s ; u, x)=\frac{1}{2 \pi} \int_{\mathbb{R}} \Psi_{A, \varepsilon}(-\lambda, x) e^{-s \lambda^{2}} e^{i \lambda u} d \lambda$.

2) The function $(u, x) \mapsto W_{\varepsilon}(s ; u, x)$ is of class $C^{1}$ on $\mathbb{R}^{2}$.

3) $\left(\Lambda_{A, \varepsilon}+\partial_{u}\right) W_{\varepsilon}(s ; u, x)=0$.

4) $\lim _{\|(u, x)\| \rightarrow+\infty} W_{\varepsilon}(s ; u, x)=0$.

Proof. 1) For $x=0$, we have $W_{\varepsilon}(s ; u, 0)=p_{s}(u, 0)=\frac{e^{-u^{2} / 4 s}}{2 \sqrt{\pi s}}$. Thus, for $x=0$, the statement follows from the well known fact

$$
\int_{\mathbb{R}} e^{-s \lambda^{2}} e^{i \lambda \xi} d \lambda=\sqrt{\frac{\pi}{s}} e^{-\frac{\xi^{2}}{4 s}}
$$

For $x \neq 0$, using again (4.18) together with the Laplace type representation (4.7) of $V_{A, \varepsilon}$, we have

$$
\begin{aligned}
W_{\varepsilon}(s ; u, x) & =\frac{1}{2 \pi} \int_{-|x|}^{|x|} \mathbb{K}_{\varepsilon}(x, y)\left(\int_{\mathbb{R}} e^{-s \lambda^{2}} e^{i \lambda(u-y)} d \lambda\right) d y \\
& =\frac{1}{2 \pi} \int_{\mathbb{R}}\left(\int_{-|x|}^{|x|} \mathbb{K}_{\varepsilon}(x, y) e^{-i \lambda y} d y\right) e^{-s \lambda^{2}} e^{i \lambda u} d \lambda \\
& =\frac{1}{2 \pi} \int_{\mathbb{R}} \Psi_{A, \varepsilon}(-\lambda, x) e^{-s \lambda^{2}} e^{i \lambda u} d \lambda .
\end{aligned}
$$

2) For $|x| \geq x_{0}$ with $x_{0}>0$, the statement follows from 1) and the growth estimate of $\left|\partial_{x} \Psi_{A, \varepsilon}(\lambda, x)\right|$ (see Theorem 3.5). Assume that $|x| \leq x_{0}$. Using the fact that

$$
\left|\varphi_{\mu}^{\prime}(x)\right| \leq c\left|\mu^{2}+\varrho^{2}\right|(|x|+1)|x| e^{(|\operatorname{Im} \mu|-\varrho)|x|},
$$

and that

$$
\left|\varphi_{\mu}^{\prime \prime}(x)\right| \leq c\left|\mu^{2}+\varrho^{2}\right|(|x|+1)^{2} e^{(|\operatorname{Im} \mu|-\varrho)|x|}
$$


for all $\mu \in \mathbb{C}$ and $x \in \mathbb{R}$ (cf. [33, Proposition 6.I.5]), we deduce from (3.3) that

$$
\left|\partial_{x} \Psi_{A, \varepsilon}(\lambda, x)\right| \leq c(|\lambda|+1)^{2}(|x|+1)^{2} e^{\left(\left|\operatorname{Im} \mu_{\varepsilon}\right|-\varrho\right)|x|},
$$

where $\mu_{\varepsilon}^{2}=\lambda^{2}-\left(1-\varepsilon^{2}\right) \varrho^{2}$. It follows that in both cases $\lambda^{2}-\left(1-\varepsilon^{2}\right) \varrho^{2} \gtreqless 0$, we have $\left|\partial_{x} \Psi_{A, \varepsilon}(\lambda, x)\right| \leq$ $c(|\lambda|+1)^{2}$ for all $|x| \leq x_{0}$.

3) In view of 1), the present statement is easy to check.

4) For $x=0, W_{\varepsilon}(s ; u, 0)=p_{s}(u, 0)=\frac{e^{-\frac{u^{2}}{4 s}}}{2 \sqrt{\pi s}} \rightarrow 0$ as $\|(u, x)\| \rightarrow \infty$.

For $x \neq 0$, using 1) and the growth property of the eigenfunction $\Psi_{A, \varepsilon}$ in Theorem 3.4.4, we get

$$
\left|W_{\varepsilon}(s ; u, x)\right| \leq c_{\varepsilon}(1+|x|) e^{-\varrho\left(1-\sqrt{1-\varepsilon^{2}}\right)|x|} .
$$

Now, the statement follows by means of polar coordinates.

The following lemma is also needed.

Lemma 4.12. Writing $W_{\varepsilon}$ as $W_{\varepsilon}(s ; u, x)=W_{\varepsilon}^{\mathrm{e}}+W_{\varepsilon}^{\mathrm{o}}$, as a sum of the even and the odd part of $W_{\varepsilon}$ with respect to $x$, we have $W_{\varepsilon}^{\mathrm{e}}(s ; u, x)>0$.

Proof. Using Lemma 4.11,1 together with the expression (3.4) of the eigenfunction $\Psi_{A, \varepsilon}$, we have

$$
\begin{aligned}
& W_{\varepsilon}(s ; u, x) \\
= & \frac{1}{2 \pi} \int_{\mathbb{R}}\left(\varphi_{\mu_{\varepsilon}}(x)+(-i \lambda+\varepsilon \varrho) \frac{\operatorname{sgn}(x)}{A(x)} \int_{0}^{|x|} \varphi_{\mu_{\varepsilon}}(z) A(z) d z\right) e^{-s \lambda^{2}} e^{i \lambda u} d \lambda \\
= & \frac{1}{2 \pi} \int_{\mathbb{R}} \varphi_{\mu_{\varepsilon}}(x) e^{-s \lambda^{2}} e^{i \lambda u} d \lambda+\frac{\operatorname{sgn}(x)}{2 \pi A(x)} \int_{\mathbb{R}}\left(\int_{0}^{|x|} \varphi_{\mu_{\varepsilon}}(z) A(z) d z\right)(-i \lambda+\varepsilon \varrho) e^{-s \lambda^{2}} e^{i \lambda u} d \lambda \\
= & : W_{\varepsilon}^{\mathrm{e}}(s ; u, x)+W_{\varepsilon}^{\mathrm{o}}(s ; u, x) .
\end{aligned}
$$

Next we shall prove that $W_{\varepsilon}^{\mathrm{e}}(s ; u, x)>0$. By Lemma 4.3, we have

$$
\begin{aligned}
W_{\varepsilon}^{\mathrm{e}}(s ; u, x) & =\frac{1}{\pi} \int_{0}^{+\infty} e^{-s \lambda^{2}} \cos (\lambda u)\left(\int_{0}^{|x|} K_{\varepsilon}(x, r) \cos (\lambda r) d r\right) d \lambda \\
& =\frac{1}{\pi} \int_{0}^{|x|} K_{\varepsilon}(x, r)\left(\int_{0}^{+\infty} e^{-s \lambda^{2}} \cos (\lambda u) \cos (\lambda r) d \lambda\right) d r \\
& =\frac{1}{4 \sqrt{\pi s}} \int_{0}^{|x|} K_{\varepsilon}(x, r)\left(e^{-\frac{(u-r)^{2}}{4 s}}+e^{-\frac{(u+r)^{2}}{4 s}}\right) d r .
\end{aligned}
$$

Using the fact that $r \mapsto K_{\varepsilon}(x, r)$ is even, we deduce that

$$
W_{\varepsilon}^{\mathrm{e}}(s ; u, x) \geq \frac{e^{-(|u|+|x|)^{2} / 4 s}}{4 \sqrt{\pi s}} \int_{-|x|}^{|x|} K_{\varepsilon}(x, r) d r=\frac{e^{-(|u|+|x|)^{2} / 4 s}}{2 \sqrt{\pi s}} \varphi_{i \sqrt{1-\varepsilon^{2}} \varrho}(x)>0 .
$$

Now we come to the crucial step in the proof of Theorem 4.10 .

Theorem 4.13. For every $s>0$ and $u, x \in \mathbb{R}$, we have $W_{\varepsilon}(s ; u, x) \geq 0$.

Proof. For $(u, x) \in \mathbb{R} \times\{0\}$, we have $W_{\varepsilon}(s ; u, 0)=p_{s}(u, 0)=\frac{e^{-\frac{u^{2}}{4 s}}}{2 \sqrt{\pi s}}>0$.

For $s>0$ and $(u, x) \in(\mathbb{R} \times\{0\})^{\mathrm{c}}$, assume that $W_{\varepsilon}(s ; u, x)$ is not always non-negative. Since $W_{\varepsilon}(s ; u, 0)>0$ and $\lim _{\|(u, x)\| \rightarrow+\infty} W_{\varepsilon}(s ; u, x)=0$ (see Lemma 4.11,3), then the above assumption 
implies that the function $(u, x) \mapsto W_{\varepsilon}(s ; u, x)$ admits an absolute minimum $\left(u_{0}, x_{0}\right) \in(\mathbb{R} \times\{0\})^{\mathrm{c}}$ such that $W_{\varepsilon}\left(s ; u_{0}, x_{0}\right)<0$. In particular, $W_{\varepsilon}^{\mathrm{o}}\left(s ; u_{0}, x_{0}\right)=\left(W_{\varepsilon}\left(s ; u_{0}, x_{0}\right)-W_{\varepsilon}\left(s ; u_{0},-x_{0}\right)\right) / 2 \leq 0$. We claim that

$$
W_{\varepsilon}^{\mathrm{o}}\left(s ; u_{0}, x_{0}\right)<0 .
$$

Indeed, if $W_{\varepsilon}^{\mathrm{o}}\left(s ; u_{0}, x_{0}\right)=0$, then $W_{\varepsilon}\left(s ; u_{0}, x_{0}\right)=W_{\varepsilon}^{\mathrm{e}}\left(s ; u_{0}, x_{0}\right)$, which is impossible since $W_{\varepsilon}\left(s ; u_{0}, x_{0}\right)<0$ while $W_{\varepsilon}^{\mathrm{e}}\left(s ; u_{0}, x_{0}\right)>0$ (see Lemma 4.12).

On the other hand, using the fact that $\left(u_{0}, x_{0}\right)$ is an absolute minimum, we have

$$
\begin{aligned}
\left(\Lambda_{A, \varepsilon}+\partial_{u}\right) W_{\varepsilon}\left(s ; u_{0}, x_{0}\right) & =\left(\frac{A^{\prime}\left(x_{0}\right)}{A\left(x_{0}\right)}+2 \varepsilon \varrho\right) W_{\varepsilon}^{\mathrm{o}}\left(s ; u_{0}, x_{0}\right)-\varepsilon \varrho W_{\varepsilon}\left(s ; u_{0}, x_{0}\right) \\
& =-\varepsilon \varrho W_{\varepsilon}^{\mathrm{e}}\left(s ; u_{0}, x_{0}\right)+\left(\frac{A^{\prime}\left(x_{0}\right)}{A\left(x_{0}\right)}+\varepsilon \varrho\right) W_{\varepsilon}^{\mathrm{o}}\left(s ; u_{0}, x_{0}\right) .
\end{aligned}
$$

Recall that our assumption is that $W_{\varepsilon}(s ; u, x)$ is not always non-negative for all $s>0$ and $(u, x) \in(\mathbb{R} \times\{0\})^{\mathrm{c}}$. We shall use Lemma 4.11,2 to prove that this assumption fails.

case 1: For $\varrho=0$, the identity (4.20) reads

$$
\left(\Lambda_{A, \varepsilon}+\partial_{u}\right) W_{\varepsilon}\left(s ; u_{0}, x_{0}\right)=\frac{A^{\prime}\left(x_{0}\right)}{A\left(x_{0}\right)} W_{\varepsilon}^{\mathrm{o}}\left(s ; u_{0}, x_{0}\right) .
$$

Now, Lemma 4.11.2 and the inequality (4.19) imply that $\left(A^{\prime} / A\right)\left(x_{0}\right)=0$, which is not true in the light of the hypotheses $(\mathrm{H} 2)$ and $(\mathrm{H} 4)$ on $A^{\prime} / A$ with $\varrho=0$.

case 2: Let $\varrho>0$ and $\varepsilon=0$. As in the previous case, Lemma 4.11.2 and the inequality (4.19) imply that $\left(A^{\prime} / A\right)\left(x_{0}\right)=0$. However, by the hypothesis $(\mathrm{H} 2)$ on $A^{\prime} / A$, we have $\left(A^{\prime} / A\right)(x) \gtreqless$ $\pm 2 \varrho \gtrless 0$ for all $x \gtrless 0$. Hence our assumption does not hold true.

case 3: Let $\varrho>0$ and $\varepsilon>0$.

subcase 3.1: Assume that $x_{0}>0$. As $W_{\varepsilon}^{\mathrm{e}}\left(s ; u_{0}, x_{0}\right)>0$ and $W_{\varepsilon}^{\mathrm{o}}\left(s ; u_{0}, x_{0}\right)<0$, it follows from (4.21) that $\left(\Lambda_{A, \varepsilon}+\partial_{u}\right) W_{\varepsilon}\left(s ; u_{0}, x_{0}\right)<0$, which is absurd by Lemma 4.11.2.

subcase 3.2: Assume that $x_{0}<0$. We pin down that

$$
\frac{A^{\prime}\left(x_{0}\right)}{A\left(x_{0}\right)}+2 \varepsilon \varrho \leq-2(1-\varepsilon) \varrho \leq 0 \text {. }
$$

By Lemma 4.11,2, we have

$$
\left(\Lambda_{A, \varepsilon}+\partial_{u}+\varepsilon \varrho \mathrm{id}\right) W_{\varepsilon}\left(s ; u_{0}, x_{0}\right)=\varepsilon \varrho W_{\varepsilon}\left(s ; u_{0}, x_{0}\right)<0,
$$

while, by (4.20), (4.19) and (4.22),

$$
\left(\Lambda_{A, \varepsilon}+\partial_{u}+\varepsilon \varrho \text { id }\right) W_{\varepsilon}\left(s ; u_{0}, x_{0}\right)=\left(\frac{A^{\prime}\left(x_{0}\right)}{A\left(x_{0}\right)}+2 \varepsilon \varrho\right) W_{\varepsilon}^{\mathrm{o}}\left(s ; u_{0}, x_{0}\right) \geq 0
$$

which contradicts the inequality (4.23).

case 4: Let $\varrho>0$ and $\varepsilon<0$.

subcase 4.1: Assume that $x_{0}>0$. Note that

$$
\frac{A^{\prime}\left(x_{0}\right)}{A\left(x_{0}\right)}+2 \varepsilon \varrho \geq 2(1+\varepsilon) \varrho \geq 0 .
$$

Hence, the identities (4.20), (4.19) and (4.24) imply that $\left(\Lambda_{A, \varepsilon}+\partial_{u}\right) W_{\varepsilon}\left(s ; u_{0}, x_{0}\right)<0$, which is absurd by Lemma 4.11,2.

subcase 4.2: Assume that $x_{0}<0$. On the one hand, by Lemma 4.11,2, we have

$$
\left(\Lambda_{A, \varepsilon}+\partial_{u}-\varepsilon \varrho \mathrm{id}\right) W_{\varepsilon}\left(s ; u_{0}, x_{0}\right)=-\varepsilon \varrho W_{\varepsilon}\left(s ; u_{0}, x_{0}\right)<0
$$


On the other hand, since $x_{0}<0$ we have $\left(A^{\prime} / A\right)\left(x_{0}\right)<0$. Thus, by (4.20) and (4.19),

$$
\left(\Lambda_{A, \varepsilon}+\partial_{u}-\varepsilon \varrho \mathrm{id}\right) W_{\varepsilon}\left(s ; u_{0}, x_{0}\right)=-2 \varepsilon \varrho W_{\varepsilon}^{\mathrm{e}}\left(s ; u_{0}, x_{0}\right)+\frac{A^{\prime}\left(x_{0}\right)}{A\left(x_{0}\right)} W_{\varepsilon}^{\mathrm{o}}\left(s ; u_{0}, x_{0}\right)>0,
$$

which contradicts the inequality (4.25).

This finishes the proof of Theorem 4.13 .

Now we are ready to prove the central result of this section.

Proof of Theorem 4.10 Let $f$ be a positive function in $\mathscr{D}(\mathbb{R})$. Proving that $V_{A, \varepsilon}(f) \geq 0$ is equivalent to showing that ${ }^{\mathrm{t}} V_{A, \varepsilon}(f) \geq 0$ (see (4.7) and (4.17)).

By (4.16) we have

$$
\int_{\mathbb{R}} f(x) V_{A, \varepsilon}\left(p_{s}(u, .)\right)(x) A(x) d x=\int_{\mathbb{R}}{ }^{\mathrm{t}} V_{A, \varepsilon} f(x) p_{s}(x, u) d x=\left({ }^{\mathrm{t}} V_{A, \varepsilon} f * q_{s}\right)(u),
$$

where $q_{s}(r):=\frac{e^{-r^{2} / 4 s}}{2 \sqrt{\pi s}}$ and $*$ is the Euclidean convolution product. Since $f \geq 0$ and $V_{A, \varepsilon}\left(p_{s}(u,).\right)(x)=$ $W_{\varepsilon}(s ; u, x) \geq 0$, it follows that $\left({ }^{\mathrm{t}} V_{A, \varepsilon} f * q_{s}\right)(u) \geq 0$ for all $s>0$ and $u, x \in \mathbb{R}$. Thus

$$
0 \leq \lim _{s \rightarrow 0}\left({ }^{\mathrm{t}} V_{A, \varepsilon} f * q_{s}\right)(u)={ }^{\mathrm{t}} V_{A, \varepsilon} f(u) .
$$

\section{REFERENCES}

[1] J.-Ph. Anker, F. Ayadi and M. Sifi, Opdam's hypergeometric functions: product formula and convolution structure in dimension 1, Adv. Pure Appl. Math. 3 (2012), 11-44.

[2] N. Ben Salem and A. Ould Ahmed Salem, Convolution structure associated with the Jacobi-Dunkl operator on $\mathbb{R}$, Ramanujan J. 12 (2006), 359-378.

[3] S. Ben Said, A. Boussin, and M. Sifi, Uncertainty principles and characterization of the heat kernel for certain differential-reflection operators, Adv. Pure Appl. Math. 6 (2015), no. 4, 215-239.

[4] S. Ben Said, A. Boussin, and M. Sifi, $L^{p}$-Fourier analysis associated with certain differential-reflection operators, preprint.

[5] H. Chébli, Sur un théorème de Paley-Wiener associé à la décomposition spectrale d'un opérateur de SturmLiouville sur $(0, \infty)$, J. Functional Analysis 17 (1974), 447-461.

[6] H. Chébli, Théorème de Paley-Wiener associé à un opérateur différentiel singulier sur $(0, \infty)$, J. Math. Pures Appl. (9) 58 (1979), no. 1, 1-19.

[7] I. Cherednik, A unification of Knizhnik-Zamolodchnikov equations and Dunkl operators via affine Hecke algebras, Invent. Math. 106 (1991), 411-432.

[8] F. Chouchane, M. Mili, and K. Trimèche, Positivity of the intertwining operator and harmonic analysis associated with the Jacobi-Dunkl operator on $\mathbb{R}$, Anal. Appl. (Singap.) 1 (2003), no. 4, 387-412.

[9] C. Dunkl, Differential-difference operators associated to reflection groups, Trans. Amer. Math. Society 311 (1989), 167-183

[10] C. Dunkl, Hankel transforms associated to finite reflection groups, Hypergeometric functions on domains of positivity, Jack polynomials, and applications (Tampa, FL, 1991), 123-138, Contemp. Math., 138, Amer. Math. Soc., Providence, RI, 1992.

[11] C. F. Dunkl and Y. Xu, Orthogonal polynomials of several variables, 2nd edition. Encyclopedia of Mathematics and its Applications. Cambridge University Press, Cambridge, (2014). xviii+420 pp.

[12] M. F. E. de Jeu, The Dunkl transform, Invent. Math. 113 (1993), no. 1, 147-162.

[13] L. Gallardo and K. Trimèche, Singularities and analytic continuation of the Dunkl and the Jacobi-Cherednik intertwining operators and their duals, J. Math. Anal. Appl. 396 (2012), no. 1, 70-83.

[14] L. Gallardo and K. Trimèche, Positivity of the Jacobi-Cherednik intertwining operator and its dual, Adv. Pure Appl. Math. 1 (2010), no. 2, 163 ?194. 
[15] I. S. Gradshteyn and I. M. Ryzhik, Table of integrals, series, and products, Translated from the Russian. Translation edited and with a preface by Alan Jeffrey and Daniel Zwillinger. Seventh edition. Elsevier/Academic Press, Amsterdam, 2007. xlviii+1171 pp.

[16] G. J. Heckman, Dunkl operators, Séminaire Bourbaki 828, 1996-97; Astérisque 245 (1997), $223-246$.

[17] G. J. Heckman, An elementary approach to the hypergeometric shift operators of Opdam, Invent. Math. 103 (1991), no. 2, 341-350.

[18] G.J. Heckman and E. Opdam, Root systems and hypergeometric functions. I, Compositio Math. 64 (1987), no. 3, 329-352.

[19] G. J. Heckman and H. Schlichtkrull, Harmonic analysis and special functions on symmetric spaces, Perspectives in Mathematics, 16. Academic Press, Inc., San Diego, CA, 1994. xii+225 pp.

[20] T. H. Koornwinder, Orthogonal polynomials in two variables which are eigenfunctions of two algebraically independent partial differential operators. I, II, III, IV, Nederl. Akad. Wetensch. Proc. Ser. A 77=Indag. Math. 36 (1974), 48-58, 59-66, 357-369, 370-381.

[21] J. L. Lions, Opérateurs de Delsarte et problèmes mixtes, Bull. Soc. Math. France 84 (1956), 9-95

[22] M. A. Mourou and K. Trimèche, Transmutation operator and Paley-Wiener theorem associated with a singular Differential-difference operator on the real line, Anal. Appl. 1 (2003), pp. 43-70.

[23] E. M. Opdam, Root systems and hypergeometric functions. III, IV Compositio Math. 67 (1988), 21-49, 191209.

[24] E. M. Opdam, Dunkl operators, Bessel functions and the discriminant of a finite Coxeter group Compositio Math. 85 (1993), no. 3, 333-373.

[25] E. M. Opdam, Harmonic analysis for certain representations of graded Hecke algebras, Acta. Math. 175 (1995), 75-121.

[26] M. Rosenblum, Generalized Hermite polynomials and the Bose-like oscillator calculus, Oper. Theory Adv. Appl. 73 (1994), 369-396.

[27] M. Rösler, Positivity of Dunkl's intertwining operator, Duke Math. J. 98 (1999), 445-463.

[28] M. Rösler, Bessel-type signed hypergroups on $\mathbb{R}$, In: Heyer, H., Mukherjea, A. (eds.): Probability measures on groups and related structures XI. Proceedings, Oberwolfach 1994, pp. 292-304. Singapore: World Scientific (1995).

[29] M. Rösler and M. Voit, Markov processes related with Dunkl operators, Adv. in Appl. Math. 21 (1998), no. 4, 575-643.

[30] B. Schapira, Contributions to the hypergeometric function theory of Heckman and Opdam: sharp estimates, Schwartz space, heat kernel, Geom. Funct. Anal. 18 (2008), no. 1, 222-250.

[31] M. Thyssen, Sur certains opérateurs de transmutation particuliers, Mem. Soc. Roy. Sci. Liège (5) 6 (1961), no. $3,32 \mathrm{pp}$.

[32] K. Trimèche, Transformation intégrale de Weyl et théorème de Paley-Wiener associés à un opérateur différentiel singulier sur (0, ), J. Math. Pures Appl. (9) 60 (1981), no. 1, 51-98.

[33] K. Trimèche, Generalized wavelets and hypergroups, Gordon and Breach Science Publishers, Amsterdam, 1997. xii+354 pp.

[34] K. Trimèche, Absolute continuity of the representing measures of the Dunkl intertwining operator and of its dual and applications, Adv. Pure Appl. Math. 1 (2010), no. 2, 195-222.

[35] K. Trimèche, Harmonic analysis associated with the Cherednik operators and the Heckman-Opdam theory, Adv. Pure Appl. Math. 2 (2011), no. 1, 23-46.

[36] K. Trimèche, Positivity of the transmutation operators associated with a Cherednik type operator on the real line, Adv. Pure Appl. Math. 3 (2012), no. 4, 361-376.

[37] K. Trimèche, The transmutation operators relating to a Dunkl type operator on $\mathbb{R}$ and their positivity, Mediterr. J. Math. (2014), to appear.

S. Ben Said: Institut Élie Cartan, Université de Lorraine-Nancy, B.P. 239, F-54506 Vandoeuvres-Lès-Nancy, France

E-mail address: salem. bensaid@univ-lorraine. fr

A. Boussen et M. Sifi: Université de Tunis El Manar, Faculté des Sciences de Tunis, Laboratoire d’Analyse Mathématiques et Applications LR11ES11, 2092, Tunis, Tunisie

E-mail address: asma.boussen@live.fr, mohamed.sifi@fst.rnu.tn 